\title{
The relationship between chemical defence and death feigning in the red flour beetle (Tribolium castaneum)
}

$$
\text { by }
$$

\author{
Ian McLean
}

A thesis submitted to the Faculty of Graduate Studies and Research in partial fulfillment of

the requirements for the degree of

Masters of Science

Department of Biology

\author{
Carleton University \\ Ottawa, Ontario, Canada \\ (June 2011)
}

Copyright $\mathbb{C}$ Ian McLean, 2011 


$\begin{array}{ll}\begin{array}{l}\text { Library and Archives } \\ \text { Canada }\end{array} & \begin{array}{l}\text { Bibliotheque et } \\ \text { Archives Canada }\end{array} \\ \begin{array}{l}\text { Published Heritage } \\ \text { Branch }\end{array} & \begin{array}{l}\text { Direction du } \\ \text { Patrimoine de rédition }\end{array} \\ \begin{array}{l}\text { 395 Wellington Street } \\ \text { Ottawa ON K1A ON4 } \\ \text { Canada }\end{array} & \begin{array}{l}\text { 395, rue Wellington } \\ \text { Ottawa ON K1A ON4 } \\ \text { Canada }\end{array}\end{array}$

Your file Votre reférence

ISBN: 978-0-494-83160-1

Our file Notre référence

ISBN: 978-0-494-83160-1

NOTICE:

The author has granted a nonexclusive license allowing Library and Archives Canada to reproduce, publish, archive, preserve, conserve, communicate to the public by telecommunication or on the Internet, loan, distribute and sell theses worldwide, for commercial or noncommercial purposes, in microform, paper, electronic and/or any other formats.

The author retains copyright ownership and moral rights in this thesis. Neither the thesis nor substantial extracts from it may be printed or otherwise reproduced without the author's permission.
AVIS:

L'auteur a accordé une licence non exclusive permettant à la Bibliothèque et Archives Canada de reproduire, publier, archiver, sauvegarder, conserver, transmettre au public par télécommunication ou par l'Internet, prêter, distribuer et vendre des thèses partout dans le monde, à des fins commerciales ou autres, sur support microforme, papier, électronique et/ou autres formats.

L'auteur conserve la propriété du droit d'auteur et des droits moraux qui protège cette thèse. $\mathrm{Ni}$ la thèse ni des extraits substantiels de celle-ci ne doivent être imprimés ou autrement reproduits sans son autorisation.
In compliance with the Canadian Privacy Act some supporting forms may have been removed from this thesis.

While these forms may be included in the document page count, their removal does not represent any loss of content from the thesis.
Conformément à la loi canadienne sur la protection de la vie privée, quelques formulaires secondaires ont été enlevés de cette thèse.

Bien que ces formulaires aient inclus dans la pagination, il n'y aura aucun contenu manquant.

\section{Canadä}




\begin{abstract}
Death feigning involves prey entering a state of immobility that is initiated by contact with a predator. It is a behaviour that spans across a diverse range of species, and can be influenced by many different environmental and conditional factors; yet, despite its prevalence, it has yet to be adequately studied beyond a physiological context. Indeed, while there are a number of adaptive hypotheses for the phenomenon, few have been tested.

This study is concerned with investigating a potential link between death feigning and chemical defence in Tribolium castaneum (red flour beetle), a chemically defended species that has also been shown to death feign. However, before testing the relationship between death feigning and chemical defence, I investigated the repeatability and variability of death feigning. Death feigning duration in $T$. castaneum is poorly repeatable between days, but individuals displayed a consistent trend of increased death feigning duration over successive attempts to induce it. Average death feigning duration was also found to be a useful indication of the degree to which an individual death feigns. Using this knowledge, I compared the relationship between average death feigning duration and chemical concentration for three different chemical components of $T$. castaneum, finding no evidence for any relationship between average death feigning duration and chemical concentration in $T$. castaneum.
\end{abstract}




\section{Acknowledgements}

I would like to thank a number of people for their help and support on this thesis. Firstly, I would like to thank Dr. Tom Sherratt for being one of the best supervisors imaginable. His dedication to his students is inspiring. I don't know how he finds the energy or time to deal with so many projects on the go but it leaves me in awe. There were frustrations and setbacks during my thesis and I will be forever grateful for his patience and guidance during those times. Any success I have as a scientist will be in part due to his help and guidance during this thesis.

I am grateful for the direction and support from the members of my committee: Dr. Sue Bertram and Dr. Rees Kassen. I am also appreciative of the time and understanding given by my examining committee: Dr. Jean-Guy Godin and Dr. Jayne Yack.

I am indebted to Dr. Gaetan Leclair and Dr. Peter Silk for their help in designing the chemical defence experiments, as well as for supplying space, time and resources, in their lab at the Atlantic Forestry Center in Fredericton, for me to complete the chemical defence experiments. I am also indebted to Dr. Charles Goodnight for supplying me with cultures of Tribolium castaneum, without which this thesis would not have been possible.

I would also like to thank my past and current lab and office mates for all their helpful discussions and their friendships during my thesis: Justin Carroll, Tom Hossie, Dr. Kevin Abbott, Jennette Fox, Heather Penney, Janice Ting, Dr. Rob Laird, Tim Boland, Lindsay Jackson Derraugh, Sanoji Wijenayake, Danielle Fraser, Jess Carpinone, Abdool Yasseen, and Krystle Olson. Special thanks go to Dr. Chris Hassall for all his help and discussion on all aspects of my thesis (specifically the statistics) and Richard Webster for numerous discussions on my thesis and photographing $T$. castaneum. I would also like to thank my parents for all their help and support, and Kaitie Warren for providing needed distraction and comfort at various points during my thesis. 
I would also like to thank the following friends for all their help and support during my thesis: Phil Branford, Graham Warren, Lesley Parks, Francina Jackson, Elena Kv, Rob Klein, Stephanie Grenko, Ryan Toth, Tony Stark, Roberto Canil, Jesse Brydle, Jessica Pink, Hank Pym, Aaron Stelmach, Angy Wong, Craig Melen, Mireille, Ender Wiggin, Daniel Rosenblatt, Nathan French, Hank McCoy, Sara French, Leon French, Alyssa Hubert, Steve Rogers, Elizabeth and Evan Adams, Frank Myslik, Karen Rickards, Jessica Ingram, Phil Molloy, Janet Van Dyne, Tiffany Holland, Martin \& Tash, Sophie, Life in 2D, Ali McMillan, Thor Odinson, Lauren, Matthew Addley, Andrea Cabanas, Ashley Howard, Graydon Ebert, Bruce Banner, Samantha Parker, Carley Centen, Dana Whittaker, James Howlett. I would also like to thank the Vancouver Canuck 2010-2011 team for some really exciting playoff hockey that helped me get through writing my thesis. 


\section{Table of contents}

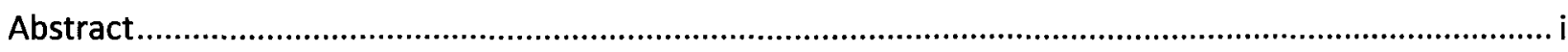

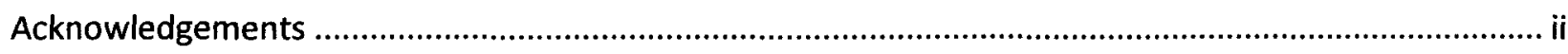

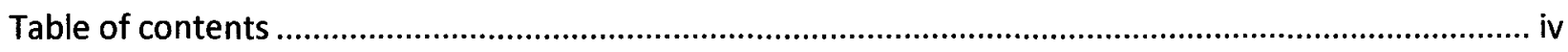

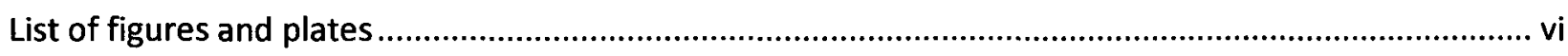

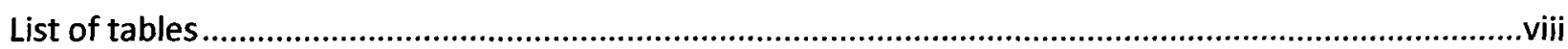

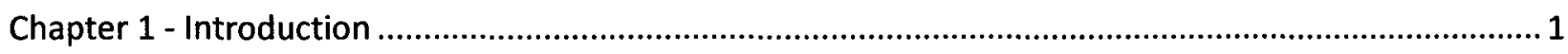

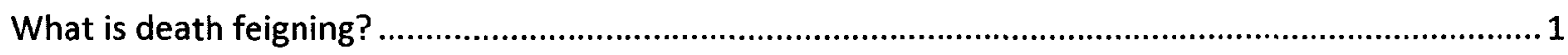

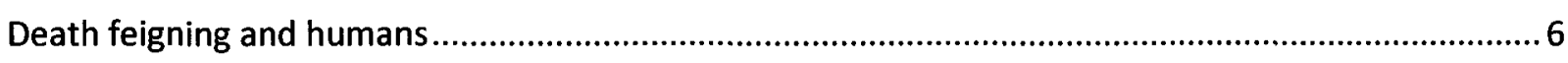

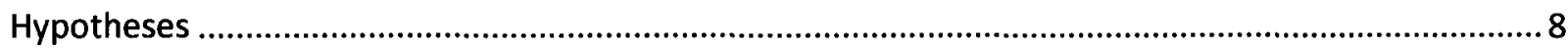

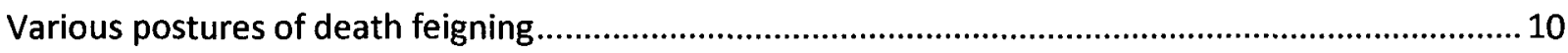

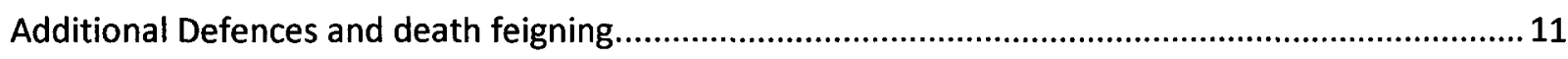

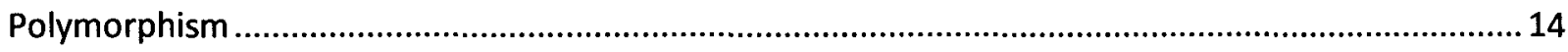

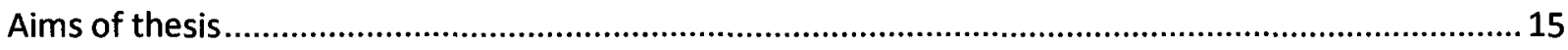

Chapter 2 - Repeatability and variability of death feigning behaviour in Tribolium castaneum .............. 17

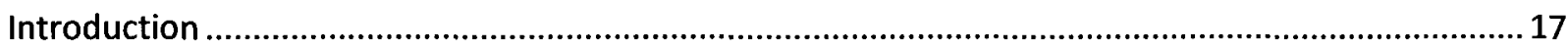

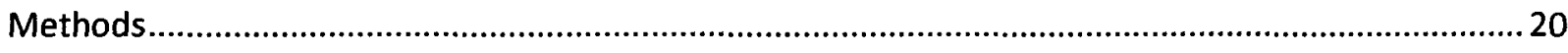

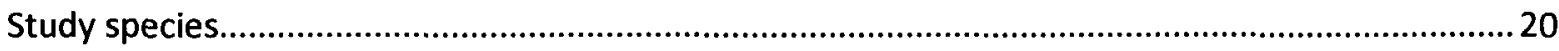

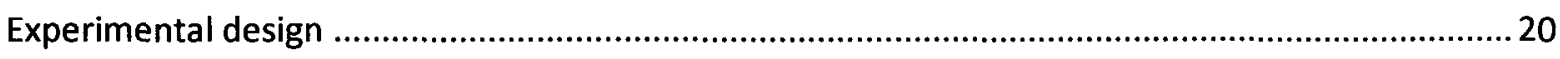

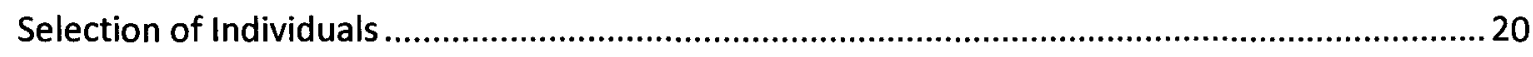

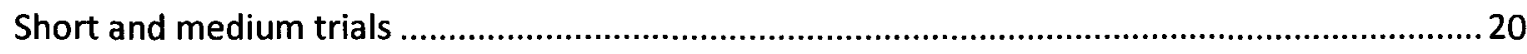

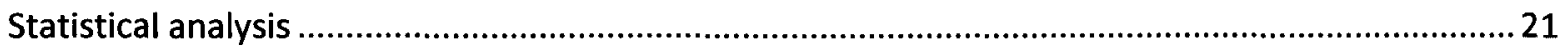

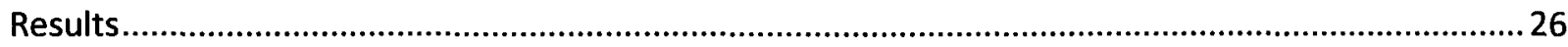

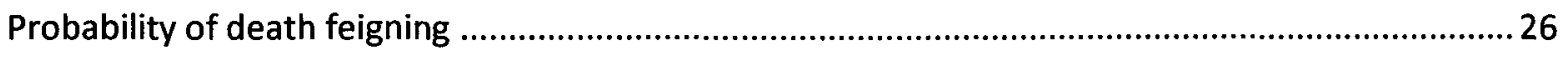

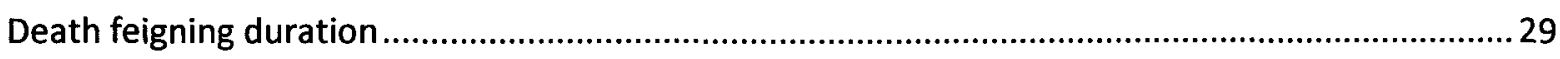

Average death feigning duration and probability of death feigning ...............................................37

Average death feigning duration and average number of taps.................................................. 37

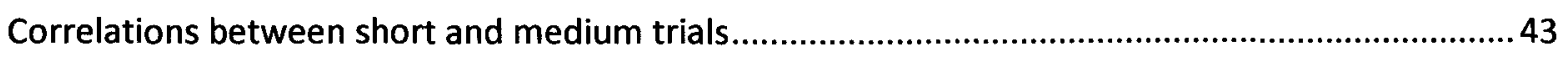

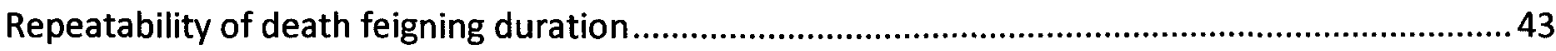


Discussion

Chapter 3 - The relationship between chemical defence and death feigning in Tribolium castaneum ..... 51

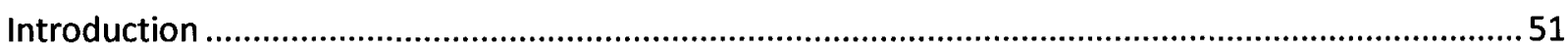

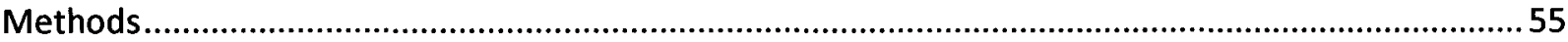

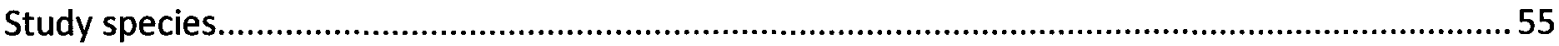

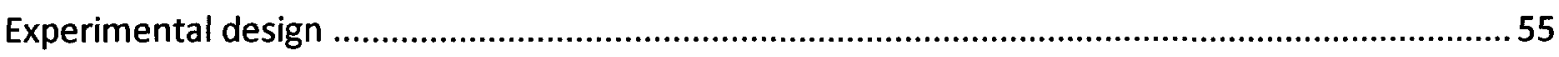

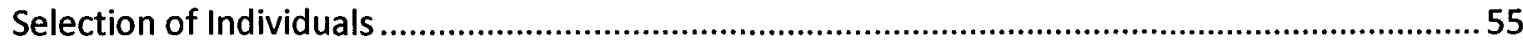

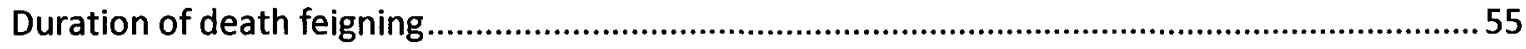

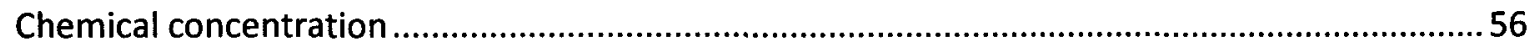

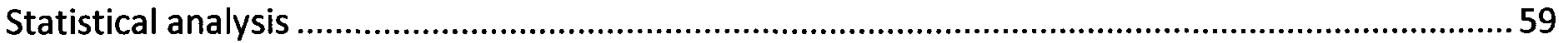

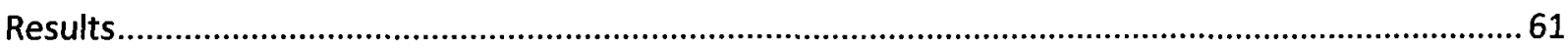

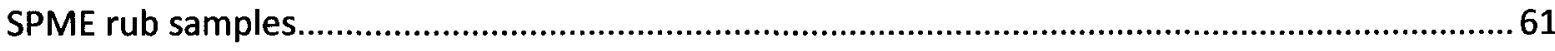

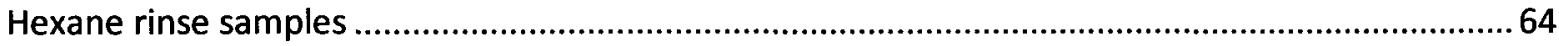

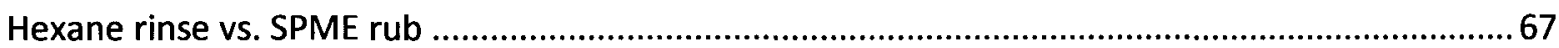

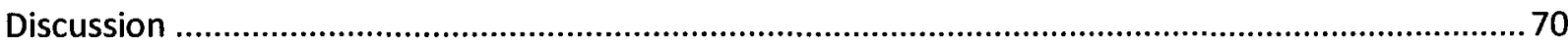

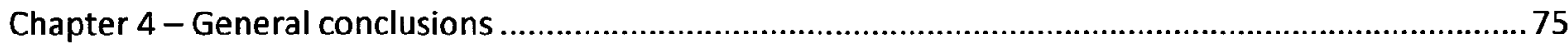

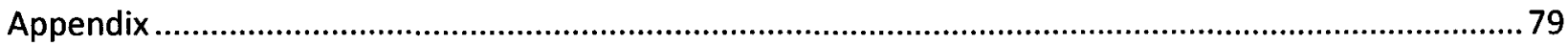

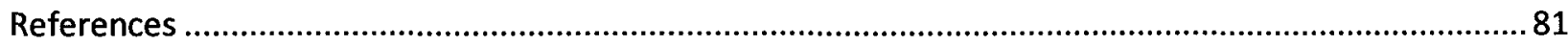

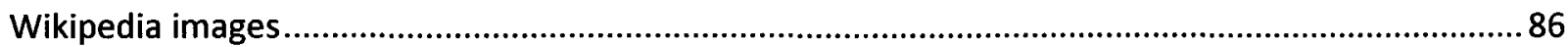




\section{List of figures and plates}

\section{Chapter 1}

Plate 1. Several representative pictures of species that death feign. Clockwise from top left: Grass snake (Natrix natrix), Opossum (Didelphis marsupialis), Red flour beetle (Tribolium castaneum), Convict tang (Acanthurus triostegus), Salamander (Bolitoglossa sp.), Mallard duck (Anas platyrhynchos). All photos taken from Wikipedia (see references).

Plate 2. Two different death feigning postures in $T$. castaneum. In the top picture, the individual has shrunk in size with contracted limbs. In the middle picture, the individual has limbs spread out wide. In the bottom picture, the individual is dead.

\section{Chapter 2}

Figure 1. Illustration of the short term death feigning testing. ............................................................22

Figure 2. Illustration of the medium term death feigning testing. ......................................................23

Figure 3. The probability of death feigning versus $A$ ) the number of attempts for the short term trials and $B$ ) the number of days for the medium term trials (error bars are $95 \%$ confidence intervals). From left to right: $\mathrm{C}++, \mathrm{BB}, \mathrm{Croatia}$. The blue circles are females and the green circles are males. 28

Figure 5. Differences in death feigning durations between the sexes for the different populations in the short trials. 32

Figure 6. Differences in death feigning durations (in seconds) between populations in the short trials. . 34

Figure 7. Differences in death feigning durations (in seconds) between populations for each age group in the short trials. From left to right: age groups (in weeks) 7-14. Within each age group, 1-3 are C++, BB and Croatia, respectively.

Figure 8. Differences in death feigning durations (in seconds) between the populations for the medium trial.

Figure 9. The average duration of death feigning (in seconds) versus A) the probability of death feigning for the short term trials and $B$ ) the probability of death feigning for the medium term trials. From left to right: $\mathrm{C}++, \mathrm{BB}, \mathrm{Croatia}$. The blue circles are females and the green circles are males.

Figure 10. Average death feigning durations (in seconds) for the sexes for the different populations in the short trials

Figure 11. Average death feigning duration (in seconds) for the populations in the medium trials. ........ 41

Figure 12. The average duration of death feigning (in seconds) versus $A$ ) the average number of taps required to induce death feigning in the short term trials and $B$ ) the average number of taps required to 
induce death feigning in the medium term trials. From left to right: $\mathrm{C}++, \mathrm{BB}, \mathrm{Croatia}$. The blue circles are females and the green circles are males.

Figure 13. Spearman's rank correlations between individuals in the short trials and the medium trials. A) Correlations between individuals for average death feigning durations (s); B) Correlations between individuals for probabilities of death feigning.

\section{Chapter 3}

Figure 1. Illustration of the method for obtaining the average death feigning duration for comparison with chemical concentrations.

Figure 2. The relationships between chemical concentration (peak area of a GC plot) and the average death feigning duration (measured in seconds). The individuals who did not emit any chemical were excluded. From top to bottom: MBQ (LN transformed), EBQ (LN transformed), PENT (square root transformed)

Figure 3. The relationship between the PCA of all the chemical components from the rubbed trials (only individuals who emitted all chemicals) and the average death feigning duration (measured in seconds).

Figure 4. The relationship between the hexane rinsed chemical concentrations (peak area of a GC plot) and the average death feigning duration (measured in seconds). From top to bottom: MBQ, EBQ, PENT.

Figure 5. The relationship between the PCA of all the chemical components from the hexane rinsed trials and the average death feigning duration (measured in seconds). .66

Figure 6. Relationships between the rinse chemical concentrations and the rub chemical concentrations for all individuals. From top to bottom: MBQ, EBQ, PENT.

Figure 7. Relationship between the PCA analysed rinse samples and the PCA analysed rub samples for all individuals. 


\section{List of tables}

\section{Chapter 1}

Table 1. The effects of different environmental and life history traits on the duration and frequency of death feigning. 4

Table 2. Several differing hypotheses for the existence of death feigning. 9

Table 3. Several examples of species that have additional defences in conjunction with death feigning. 13

\section{Chapter 2}

Table 1. Binomial logistic regression statistics, and the relationships between the probability of death feigning and several factors for the short and medium trials.

Table 2. Repeated measures generalized linear mixed effect model statistics, and the relationships between death feigning duration and several factors for the short and medium trials. Restricted likelihood ratio tests (RLRT) were used to calculate if there was a significant difference in death feigning

durations between individuals. 30

Table 3. Relationships between death feigning duration and three factors within populations for the short and medium trials.

Table 4. Relationship between average death feigning duration and several factors in the short and medium trials.

Table 5. Spearman's rank correlations testing the correlation in death feigning duration values between subsequent attempts in the short term trial. Tests were done for each sex in each population.

Table 6. Intraclass correlation coefficients for each sex in each population for the medium term trial. .. 46 


\section{Chapter 1 - Introduction}

\section{What is death feigning?}

For my thesis project, I will be examining death feigning. This is an umbrella term for a variety of phenomena (otherwise known as thanatosis, death shamming, tonic immobility, quiescence, playing possum, playing dead) that are generally associated with remaining motionless in the presence of predators. The term death feigning gives the impression that the subject is pretending to be dead, and is therefore mimicking the posture of a deceased member of that species. However, as Charles Darwin (1883) noted when studying several insect species, "in several instances the attitude of the feigners and of the really dead were as unlike as they possibly could be." Following Darwin's reservations, when I discuss death feigning for this project, it will refer to a form of immobility that is entered into for a period of time by a prey species in response to an imminent attack by a predatory species, and is thus a secondary defence (a defence that is used when a predator has the prey in its grasp) against predation (Edmunds 1974). It will not necessarily represent a perfect mimicry of death, and it will not necessarily be a voluntary behaviour.

There are three scenarios that commonly lead to a behaviour that has been labelled as death feigning. These scenarios are: 1) when a prey becomes motionless after being attacked by a predator; 2) when a predator remains motionless to avoid detection by a prey; 3) when a conspecific remains motionless as a prelude to mating (usually in situations involving precopulatory cannibalism). As noted above, I shall be treating examples of the first scenario as death feigning, and this behaviour will be the central focus of my thesis. The second scenario occurs when the predator voluntarily and consciously remains immobile in order to lure a prey 
within striking distance, as demonstrated by a predatory cichlid Haplochromis livingstoni (McKaye, 1981). The third scenario is typified in Pisaura mirabilis (the gift giving spider) and involves the male spider presenting the female spider a gift, while entering an immobile state. The female spider takes the gift, while the male slowly moves under the female and copulates with her. If the male were to move, the female would consume him before copulation (Bilde et al. 2006; Hansen et al. 2008).

Death feigning has been demonstrated in a host of species across taxa (Plate 1), including in mammals (Holmes 1916; Francq 1969; Oakley and Plotkin 1977), birds (Sargeant and Eberhardt 1975; Caro 2005), reptiles (Gregory et al. 2007; Gerald 2008), amphibians (Brodie et al. 1974; Shaffer 1978), fish (Howe 1991), and insects (Edmunds 1976; Acheampong and Mitchell 1997; Fu et al. 2006). Despite its wide appearance across taxa, it has yet to be adequately studied using an adaptive framework (Miyatake et al. 2004). The environmental conditions under which death feigning is more likely to arise in a given species have been documented, and remarks have been made about the life-histories most commonly associated with death feigning (Table 1). However, it is currently unclear whether there are any universal properties or generalisations that can be made and its adaptive significance remains unclear. 


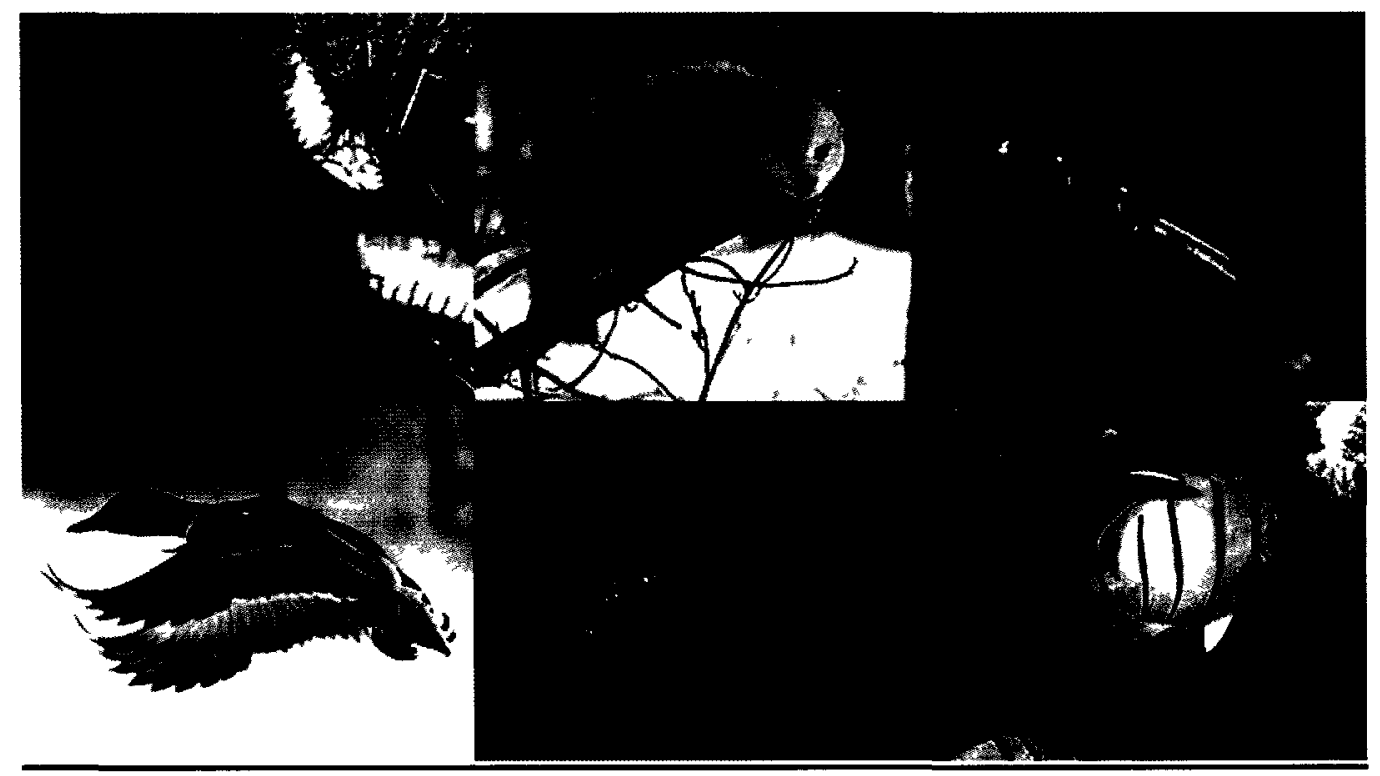

Plate 1. Several representative pictures of species that death feign. Clockwise from top left: Grass snake (Natrix natrix), Opossum (Didelphis marsupialis), Red flour beetle (Tribolium castaneum), Convict tang (Acanthurus triostegus), Salamander (Bolitoglossa sp.), Mallard duck (Anas platyrhynchos). All photos taken from Wikipedia (see references). 
Table 1. The effects of different environmental and life history traits on the duration and frequency of death feigning.

\begin{tabular}{|c|c|c|}
\hline $\begin{array}{l}\text { Effect of environmental and } \\
\text { life history traits on death } \\
\text { feigning }\end{array}$ & Species & Reference \\
\hline $\begin{array}{l}\text { Death feigning presence } \\
\text { dependent on age; some } \\
\text { species develop it when } \\
\text { younger, while others develop } \\
\text { it when older }\end{array}$ & $\begin{array}{l}\text { Opossums (Didelphis } \\
\text { marsupialis); Fire ant } \\
\text { (Solenopsis invicta) }\end{array}$ & $\begin{array}{l}\text { Francq 1969; Cassill et al. } \\
2008\end{array}$ \\
\hline $\begin{array}{l}\text { Death feigning may cease } \\
\text { after consecutive stimulations, } \\
\text { however it may increase in } \\
\text { duration in those that maintain } \\
\text { it }\end{array}$ & $\begin{array}{l}\text { Opossum (Didelphis } \\
\text { marsupialis); Colorado potato } \\
\text { beetle (Leptinotarsa } \\
\text { decemlineata); Goldfish } \\
\text { (Carassius auratus) }\end{array}$ & $\begin{array}{l}\text { Francq 1969; Acheampong } \\
\text { and Mitchell 1997; Leebevre } \\
\text { and Sabourin } 1977\end{array}$ \\
\hline $\begin{array}{l}\text { Does not always require tactile } \\
\text { stimulation from a predator }\end{array}$ & $\begin{array}{l}\text { Opossum (Didelphis } \\
\text { marsupialis); Colorado potato } \\
\text { beetle (Leptinotarsa } \\
\text { decemlineata) }\end{array}$ & $\begin{array}{l}\text { Francq 1969; Couturier et al. } \\
2005\end{array}$ \\
\hline $\begin{array}{l}\text { Death feigning can be } \\
\text { dependent on body size and } \\
\text { weight; some species increase } \\
\text { duration and frequency of } \\
\text { death feigning with increased } \\
\text { size; others do not }\end{array}$ & $\begin{array}{l}\text { Brown snake (Storeria } \\
\text { dekayi); Adzuki bean beetle } \\
\text { (Callosobruchus chinensis) }\end{array}$ & $\begin{array}{l}\text { Gerald 2008; Hozumi and } \\
\text { Miyatake } 2005\end{array}$ \\
\hline $\begin{array}{l}\text { Individuals with a shorter } \\
\text { lifespan are less likely to death } \\
\text { feign than individuals with a } \\
\text { longer life span }\end{array}$ & $\begin{array}{l}\text { Adzuki bean beetle } \\
\text { (Callosobruchus chinensis) }\end{array}$ & $\begin{array}{l}\text { Hozumi and Miyatake } 2005 ; \\
\text { Nakayama and Miyatake } 2009\end{array}$ \\
\hline $\begin{array}{l}\text { Increased starvation will lead } \\
\text { to decreased death feigning } \\
\text { duration, ending with its } \\
\text { termination }\end{array}$ & $\begin{array}{l}\text { Colorado potato beetle } \\
\text { (Leptinotarsa decemlineata); } \\
\text { Sweet potato weevil (Cylas } \\
\text { formicarius) }\end{array}$ & $\begin{array}{l}\text { Acheampong and Mitchell } \\
\text { 1997; Miyatake 2001b }\end{array}$ \\
\hline
\end{tabular}


Table 1 (cont.)

\begin{tabular}{|c|c|c|}
\hline $\begin{array}{l}\text { Increased death feigning } \\
\text { (frequency and duration) when } \\
\text { exposed to light, as opposed to } \\
\text { darkness }\end{array}$ & $\begin{array}{l}\text { Sweet potato weevil (Cylas } \\
\text { formicarius); Anoles (Anolis } \\
\text { carolinensis), Geckos } \\
\text { (Hemidactylus turcicus) }\end{array}$ & $\begin{array}{l}\text { Miyatake 2001; Hennig and } \\
\text { Dunlap } 1977\end{array}$ \\
\hline $\begin{array}{l}\text { Death feigning increases as } \\
\text { activity levels or speed of } \\
\text { escape decrease }\end{array}$ & $\begin{array}{l}\text { Sweet potato weevil (Cylas } \\
\text { formicarius); Brown snake } \\
\text { (Storeria dekayi); Adzuki } \\
\text { bean beetle (Callosobruchus } \\
\text { chinensis); Red flour beetle } \\
\text { (Tribolium castaneum); } \\
\text { Damselfly larvae (Ischnura } \\
\text { elegans) }\end{array}$ & $\begin{array}{l}\text { Miyatake 2001; Gerald 2008; } \\
\text { Ohno and Miyatake } 2007 ; \\
\text { Miyatake et al. } 2008 \mathrm{~b} ; \\
\text { Gyssels and Stoks } 2005\end{array}$ \\
\hline $\begin{array}{l}\text { Those with greater duration of } \\
\text { death feigning have greater } \\
\text { fecundity, producing larger } \\
\text { eggs }\end{array}$ & $\begin{array}{l}\text { Adzuki bean beetle } \\
\text { (Callosobruchus chinensis) }\end{array}$ & Nakayama and Miyatake 2009 \\
\hline $\begin{array}{l}\text { Death feigning individuals } \\
\text { develop faster }\end{array}$ & $\begin{array}{l}\text { Adzuki bean beetle } \\
\text { (Callosobruchus chinensis) }\end{array}$ & Nakayama and Miyatake 2009 \\
\hline $\begin{array}{l}\text { Increased time spent } \\
\text { copulating decreases duration } \\
\text { of death feigning }\end{array}$ & $\begin{array}{l}\text { Sweet potato weevil (Cylas } \\
\text { formicarius) }\end{array}$ & Kuriwada et al. 2009 \\
\hline $\begin{array}{l}\text { Death feigning has been } \\
\text { shown to increase and } \\
\text { decrease in duration and } \\
\text { frequency with increasing } \\
\text { temperature, depending on } \\
\text { species }\end{array}$ & $\begin{array}{l}\text { Adult seed beetle } \\
\text { (Callosobruchus maculates; } \\
\text { Callosobruchus chinensis); } \\
\text { Neonate brown snake } \\
\text { (Storeria dekayi) }\end{array}$ & $\begin{array}{l}\text { Miyatake et al. 2008; Gerald } \\
2008\end{array}$ \\
\hline
\end{tabular}




\section{Death feigning and humans}

Humans have also been known to experience death feigning behaviours, most often in response to a threatening situation. For example, there are countless stories of individuals having to be removed from the front lines during the first and second world wars because they had unconsciously entered into an immobile state and could no longer function as a soldier (Cantor 2005). While some of those cases may be suspect, with individuals pretending to be immobile so that they would be removed from battle, there are still a great number that were likely authentic cases where individuals had no choice in the matter (Cantor 2005). Other studies (Marks 1987, Cantor 2005, Abrams 2008) have shown it to occur quite often in cases of rape, attack by predator (animal or human), motor vehicle accidents, etc.

Interestingly, there is evidence that individuals who have experienced a traumatic event, and who entered into a state of immobility during said event, can relapse into a state of immobility when exposed to environmental triggers. For example, a young woman entered into a immobile state when she was threated during a robbery at a café. Approximately a year later, she was confronted by an aggressive acquaintance, and relapsed into an immobile and speechless state for approximately two minutes, during which time she had flashbacks of the robbery from the previous year (Cantor 2005). Death feigning in humans has been linked with posttraumatic stress disorder and can often be one of the associated symptoms; a trait seen in war survivors, rape survivors and other individuals who have survived traumatic and threatening situations (Cantor 2005).

Besides unconsciously exhibiting death feigning behaviour in a threatening situation, humans have also found ways to exploit said behaviour (personally and in other species) for 
personal benefit or societal gain. As previously mentioned, there are stories of people intentionally death feigning to be released from war or to protect themselves in a threatening situation (Cantor 2005). For example, in the 2008 Mumbai terrorist attacks, an actor from Montreal who had been wounded took advantage of his injuries and pretended to be dead, thereby fooling the terrorist gun men (Milewski 2008). There are also countless stories of people, who when encountering wild animals (particularly bears), have death feigned to avoid attack (whether this actually works is another story) (Bryson 1998).

Additionally, humans have used their knowledge of death feigning in other species to their benefit. For example, some shark species are known to death feign when they are turned onto their back, a behaviour that is thought to be involved in mating (Whitman et al. 1986). Researchers have used this death feigning behaviour when doing scientific research as a means to sample individuals for various observations, as well as to test shark repellents that may activate the death feigning behaviour (Watsky and Gruber 1990). Researchers in pest management have also benefited from knowledge about death feigning in pest species. For example, research was done on the effects of the sterile insect technique (exposing males to high levels of sterility inducing radiation) and the effect it has on death feigning in Cylas formicarius (the sweetpotato weevil) (Kuriwada et al. 2010). The intention of the sterile insect technique is for sterile males to mate with females, ultimately decreasing the number of offspring per capita. They found, however, that the radiation was causing individuals to death feign less, which increased the probability of being killed by predators. As a result, it allowed researchers to better plan when the males were subjected to the radiation, as well as when they were released to ensure the highest possible mating success. A final example concerns death feigning in livestock and domestic animals. Livestock have shown a tendency to death feign when placed in stressful 
situations (i.e. shearing, castration, herding, etc.) (Forkman et al. 2007). There is also evidence to show that animals more prone to fear responses and death feigning produce inferior meat to those who are less likely to death feign (Remignon et al. 1998). As a result, knowledge and study of death feigning in livestock would allow for farmers to artificially select for lower death feigning, while also adapting management techniques to minimize the amount of stress that the animals encounter.

\section{Hypotheses}

There are several different ultimate (as opposed to proximate) hypotheses as to why death feigning occurs (Table 2) - including "avoid the dead", "take the others" and "I'm chemically loaded" (my own terms). While these hypotheses make a degree of sense, they have generally been proposed without any direct evidence to support them, and include explanations of questionable merit (Ruxton 2006). For example, it has been suggested (Edmunds 1974) that death feigning may cause predators simply to lose interest in the prey and abandon them (just as a cat will play with an active mouse but will abandon a dead or inactive one). Rather than sheer disinterest in immobile objects, perhaps a more satisfactory hypothesis for the same observations may be that the predator assumes that the immobile prey is dead and decides to leave it for later while the predator chases after other individual prey that are escaping (Ruxton et al. 2004). Under these conditions, the predator would be trying to maximize the amount of prey it can consume, while the prey would be tricking the predator into leaving it aside, allowing for it to escape. 
Table 2. Several differing hypotheses for the existence of death feigning.

\begin{tabular}{|l|l|l|}
\hline $\begin{array}{l}\text { Hypothesis Name (names } \\
\text { are proposed by me) }\end{array}$ & Description & Reference \\
\hline Avoid the dead & $\begin{array}{l}\text { A prey entering an immobile } \\
\text { state will trick the predator } \\
\text { into thinking it is dead, and } \\
\text { the predator will not wish to } \\
\text { consume it because of the } \\
\text { potential for sickness }\end{array}$ & $\begin{array}{l}\text { Frost 1942; Miyatake et al. } \\
2009\end{array}$ \\
\hline Motion blindness & $\begin{array}{l}\text { A prey entering an immobile } \\
\text { state will cease to be a target } \\
\text { for the a predator that is } \\
\text { attracted to movement }\end{array}$ & $\begin{array}{l}\text { Edmunds 1974; Prohammer } \\
\text { and Wade 1981; Miyatake } \\
\text { 2001 }\end{array}$ \\
\hline Take the others & $\begin{array}{l}\text { A prey entering an immobile } \\
\text { state will trick the predator } \\
\text { into believing they are dead, } \\
\text { causing the predator to } \\
\text { abandon them for later, and } \\
\text { pursue other individuals }\end{array}$ & Ruxton et al. 2004; Caro 2005 \\
\hline Don't hit me & $\begin{array}{l}\text { Some species will enter an } \\
\text { immobile display in front of } \\
\text { older and more aggressive } \\
\text { neighbours of the same } \\
\text { species to avoid attack }\end{array}$ & Cassill et al. 2008 \\
\hline You can't eat me & $\begin{array}{l}\text { A prey will enter an immobile } \\
\text { state to signal to a predator } \\
\text { that it is chemically defended }\end{array}$ & $\begin{array}{l}\text { Ruxton 2006 } \\
\text { state, changing their posture in } \\
\text { a way that prevents a gap } \\
\text { limited predator from } \\
\text { consuming them }\end{array}$ \\
\hline
\end{tabular}




\section{Various postures of death feigning}

Death feigning does not always take a consistent posture in species that do it. Toledo et al. (2010) provided evidence of two distinct postures that are often both classified as death feigning. They found that some frog species would pull their limbs in close to their bodies, keep their eyes open and remain motionless, while other frog species would lay motionless with their limbs extended haphazardly and their eyes closed. They remarked that the second behaviour was a better description of death feigning (with the frogs appearing dead), while the first behaviour was more of a shrinking/contracting behaviour. Interestingly, they found that the death feigning behaviour was more likely to occur in non-toxic species, while the shrinking/contracting behaviour was more likely to occur in toxic individuals. It was reasoned that those individuals that are not toxic should take on a death feigning posture in the hopes of dissuading the predators from consuming them, while toxic individuals should shrink in the hopes of incurring minimum damage while being consumed by predators. Once consumed by predators, the toxic species could release toxins that would cause the predator to become ill and disgorge the prey, fully alive and intact (albeit with slightly eroded skin) (Sazima 1974, Toledo et al. 2010).

Slight movements of the extremities while death feigning is another variation that has been demonstrated (Francq 1969; Beux dos Santos et al. 2010). This behaviour usually occurs after the individual has been death feigning for some time, and could potentially be a means of testing or verifying whether a threat remains in the vicinity without attracting that threat. Additionally, there is evidence for death feigning in conjunction with limb autotomy (Fleming et al. 2007). Stick insects engage in limb autotomy in the presence of predators, releasing their leg(s), which will move once unattached and distract predators from the rest of their body. By 
death feigning after limb autotomy, they further increase their chances of survival by ensuring that the movement of the removed limb will be the only movement seen by the predator and thus, a more attractive target (at least for predators who hunt based on movement).

Interestingly, I witnessed some of these differences within my own study species, Tribolium castaneum. I found that some individuals displayed a shrinking behaviour, while others had limbs that were spread out (Plate 2). Additionally, I found some individuals who remained motionless except for slight but constant twitches in one of their limbs. I also found that many of the individuals performed slight movements with their extremities before exiting a death feigning state. An interesting area for future study could be an examination of the frequency of these different immobile postures, and potential relationships with levels of toxicity or other life history factors.

\section{Additional Defences and death feigning.}

Having reviewed the literature, I've discovered a number of cases where death feigning is accompanied with an additional defence (although this is not reported in all species (Holmes 1916; Sargeant and Eberhardt 1975; Toledo et al. 2010) (Table 3). An association between the two traits (death feigning and chemical defence) has recently been speculated (Miyatake et al. 2004, Ruxton 2006), and examined (Miyatake et al. 2009), however, no one has yet provided direct evidence to support this, or explore its implications. I feel that this is an important area to examine. As noted above, entering an immobile state may act as an honest signal of chemical defence (Ruxton 2006). A second possibility may be that an additional defence cannot be activated while moving, and that entering a state of immobility allows for the release of a chemical or the movement of a spine. 

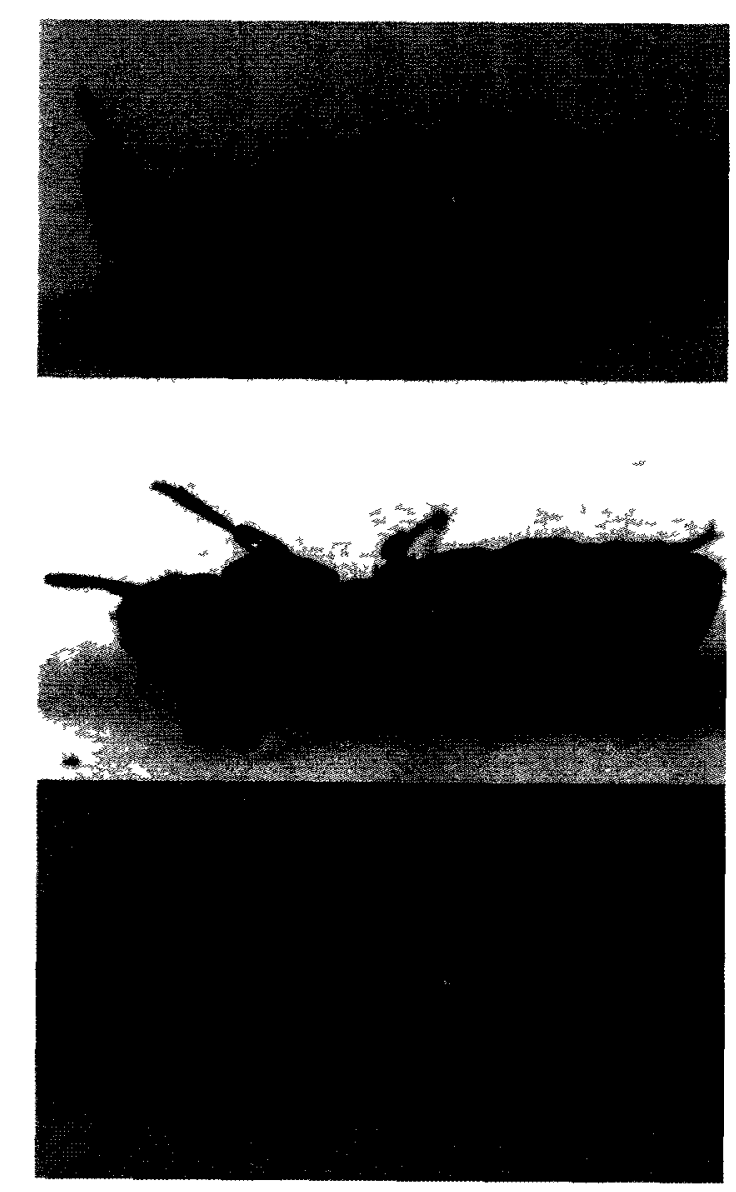

Plate 2. Two different death feigning postures in T. castaneum. In the top picture, the individual has shrunk in size with contracted limbs. In the middle picture, the individual has limbs spread out wide. In the bottom picture, the individual is dead. 
Table 3. Several examples of species that have additional defences in conjunction with death feigning.

\begin{tabular}{|l|l|l|}
\hline Species & Defence & Reference \\
\hline $\begin{array}{l}\text { Red flour beetle (Tribolium } \\
\text { castaneum) }\end{array}$ & Noxious chemical release & Miyatake et al. 2004 \\
\hline $\begin{array}{l}\text { Three species of } \text { Allothyrus } \\
\text { mites }\end{array}$ & $\begin{array}{l}\text { Retraction of limbs close to } \\
\text { body that is covered with } \\
\text { body armour; noxious } \\
\text { chemical release }\end{array}$ & Walter and Proctor 1998 \\
\hline $\begin{array}{l}\text { Aquatic firefly larvae (Luciola } \\
\text { leii) }\end{array}$ & $\begin{array}{l}\text { Noxious chemical release; } \\
\text { luminescence (may act as } \\
\text { deterring signal of toxicity) }\end{array}$ & Fu et al. 2007 \\
\hline $\begin{array}{l}\text { Adult firefly (Pyrocoelia } \\
\text { pectoralis) }\end{array}$ & $\begin{array}{l}\text { Noxious chemical release; } \\
\text { luminescence (may act as } \\
\text { deterring signal of toxicity) }\end{array}$ & Fu et al. 2006 \\
\hline $\begin{array}{l}\text { Opossum (Didelphis } \\
\text { marsupialis) }\end{array}$ & $\begin{array}{l}\text { Foul smelling green discharge } \\
\text { from anus }\end{array}$ & Francq 1969 \\
\hline $\begin{array}{l}\text { Pygmy grasshopper (Criotettix } \\
\text { japonicas) }\end{array}$ & Lateral spines & Honma et al. 2006 \\
\hline
\end{tabular}




\section{Polymorphism}

In some examples (although not all - see Sargeant and Eberhardt 1975) death feigning is considered to be a polymorphic trait within a population, in that some individuals engage in it and others do not (Prohammer and Wade 1981). Death feigning is a heritable trait, with frequencies that vary between populations (Prohammer and Wade 1981; Miyatake et al. 2004). As shown in Table 1, there are several different environmental factors which can affect both the duration and initiation of death feigning in an individual (Miyatake et al. 2004). While these factors can certainly influence the frequency of death feigning, the heritable nature of the trait suggests that they cannot be the sole factors generating the within-population variability. Prohammer and Wade (1981) discovered that a population of Tribolium castaneum cultured for over 100 generations in lab, without any artificial selection for death feigning, had a frequency of death feigning approaching $0 \%$. As these beetles were treated under the same environmental conditions as four other geographically distinct populations, which had varying death feigning frequencies, it raises the possibility that a death feigning polymorphism may be maintained by other selective pressures, including frequency-dependent selection.

While selection on death feigning may be frequency-dependent within populations, it may also vary geographically as a result of variation in the intensity of selection. Honma et al. (2006) described a species of grasshopper, that entered an immobile state in the presence of a specific frog predator; an action that was not repeated with any other predator. So, death feigning could be maintained in a polymorphic equilibrium by specific predators in any given locale, but this equilibrium can be different in different areas, and may even be free to drift in populations which are devoid of specific predators. 


\section{Aims of thesis}

Death feigning, despite being prevalent in numerous species across, is a subject that has not been thoroughly researched or examined within an adaptive framework. It is my hope to quantify some of the relationships that have been tentatively proposed in previous research.

The main goal of my thesis was to investigate whether or not there is a relationship between death feigning and chemical defence, using $T$. castaneum (a species that death feigns and has a chemical defence) as the model species. I wanted to verify whether or not $T$. castaneum death feigns because of chemical defence or whether additional hypotheses are required to explain death feigning in $T$. castaneum. To date, there has only been one study, that I am aware of, to address this question (Miyatake et al. 2009), and I feel that it was somewhat inadequate, as it only examined one chemical component of the $T$. castaneum defence secretion and only examined males. Using two different methods for testing chemical concentration, I examined three of the principal chemical components that make up the $T$. castaneum defensive secretion, in both males and females.

However, before I could begin to test the relationship between death feigning and chemical defence, I first needed to understand more about death feigning behaviour in my $T$. castaneum populations. Do they all death feign? Can I categorize individuals into death feigners and non-death feigners? If an individual death feigns today, will they death feign tomorrow? Additionally, I needed to identify a reliable measure for how much of a death feigner an individual was, for use in testing the relationship between chemical defence and death feigning. To do this, I examined how an individual's propensity to death feign changed over a short term trial, that involved multiple attempts to induce death feigning within a single day, and a medium 
term trial, that involved multiple attempts to induce death feigning spread over five consecutive days.

I feel that this thesis addresses an important unresolved question concerning the evolution of death feigning in $T$. castaneum and its relationship to chemical defence, while also providing novel information on death feigning behaviour in T. castaneum. Additionally, as research on the evolutionary and adaptive significance of death feigning has been somewhat limited, it is my hope that this thesis will raise awareness about an important and prevalent animal behaviour. 


\section{Chapter 2 - Repeatability and variability of death feigning behaviour in Tribolium castaneum}

\section{Introduction}

Death feigning is a secondary anti-predator defence, wherein the individual enters a state of immobility after contact with a predator (Edmunds 1974). It is a behaviour seen in a wide range of species across taxa, however, despite its widespread presence, research into the ultimate causes of death feigning is nascent and ongoing (Miyatake et al. 2004). Before research can be done on the ultimate causes of death feigning and how death feigning relates to other life history traits in different species, it is important to understand the intra-species variability in death feigning, including how repeatable the behaviour is, which has not been studied in great detail.

Repeatability is important to consider in death feigning experiments in providing context for any given observation. There is a tendency by researchers to assume that death-feigning is time invariant and effectively a binary $(1 / 0)$ response. However, if an individual is labelled as a "death feigner" and used in an experiment comparing death feigners and non-death feigners, the results may be questionable if the propensity to death-feign is a continuous rather than discrete trait. Thus knowing, for example, whether an individual who death feigned yesterday will death feign tomorrow is an important but usually overlooked aspect.

There are a few studies that have looked at repeatability and intra-species variability in death feigning behaviour and most of it has centred on death feigning duration rather than death feigning propensity. Watsky and Gruber (1990) found that death feigning duration in juvenile lemon sharks (Negaprion brevirostris) decreased over successive attempts to induce death feigning, with individuals becoming more habituated over multiple successive attempts in one 
day, and in single attempts spread out over multiple days. Studies done on domestic chicks over a four day period found that death feigning duration likewise decreased over the days, indicating habituation (Jones 1977). Individual hens and quail appear to reach stable death feigning durations over three days of testing (Jones 1988), indicating high repeatability (Forkman et al. 2007). There were mixed results in different groups of pigs, with some studies showing low repeatability of death feigning duration (repeatability value below 0.40 ), while another study showed high repeatability (Forkman et al. 2007). Francq (1969) examined death feigning duration in 6 opossums (Didelphis marsupialis) over a maximum of 60 days, finding that all individuals consistently death feigned at $11,23,30,33,35$ and 60 days, respectively, yet for varying amounts of time. Nakayama et al. (2009) examined repeatability of 80 individual confused flour beetles (Tribolium confusum) over two days. They found the behaviour to be highly repeatable, with a repeatability value of 0.94 .

As I mentioned in Chapter 1, I am interested in examining the relationship between death feigning and chemical defence in T. castaneum (Chapter 3). Before I can do that, however, I need to establish whether T. castaneum individuals from my populations can be classified as death feigners and non-death feigners or if I need to use an alternative method to classify individuals. Previous studies on death feigning in $T$. castaneum have either used artificially selected lines of death feigners and non-death feigners (Miyatake et al. 2004) or have found that their populations were polymorphic (Prohammer and Wade 1981). I cannot assume, however, that my populations will be similar, as I am using base populations that have not been subject to artificial selection on death feigning. Moreover, the populations I am working with have been laboratory reared for multiple generations rather than being directly taken from the wild. 
Here I examined the repeatability and variability of death feigning behaviour in three different $T$. castaneum populations. I quantified both death feigning duration and the probability of death feigning in individuals in a short term trial, with each individual exposed to five attempts to induce death feigning within a single day, and a medium term trial, with each individual exposed to five attempts to induce death feigning spread out over five days. I also investigated the repeatability of death feigning duration between days in the medium term trials, as well as examining how consistent death feigning patterns were between individuals in the short term trials. The work differs from Nakayama et al. (2009) not only because it is a different species, but also because it investigates death-feigning repeatability over two separate time scales and because it attempted to account directly for both sex and age-dependent variation. 


\section{Methods}

\section{Study species}

Three base populations $(\mathrm{C}++, \mathrm{BB}$, and Croatia) of $100 \mathrm{~T}$. castaneum individuals each were provided by Dr. Charles Goodnight of the University of Vermont, USA. C++ was a local laboratory strain (reared for over 12 years in lab, with an exact rearing time in lab unknown), while both BB (originally sampled from a site in Boynton Beach, Florida) and Croatia (originally sampled from a site in Croatia) were lab reared for approximately 12 years. Individuals in this experiment were maintained in canning jars, in a mixture of $95 \%$ whole wheat flour and $5 \%$ brewer's yeast by weight. Cultures were stored in an incubator at approximately $29^{\circ} \mathrm{C}$ on a 14 hour light cycle (8:00 to $22: 00)$.

\section{Experimental design}

\section{Selection of Individuals}

Final instar larvae and pupae were selected and separated into $1.5 \mathrm{~mL}$ microtubes, filled with $1.0 \mathrm{~mL}$ of $95 \%$ whole wheat flour and $5 \%$ brewer's yeast by weight. Microtubes were checked every week for adult eclosion. Once adults had eclosed, individuals were sexed by identification of prothoracic femoral setiferous sex patches; males have them, females do not.

\section{Short and medium trials}

Death feigning was induced in individuals, under a dissecting microscope, by tapping between the prosternum and mesosternum with metal tweezers. Death feigning duration was 
recorded with a stopwatch to the nearest second, and the number of taps required to induce death feigning was also recorded.

Between 5 and 6 weeks after eclosion, death feigning in individuals was tested in a "medium term" trial that took place over 5 days. Each day death feigning was induced in each individual. Individuals were tapped until they death feigned or until a maximum of 10 taps had been reached (Fig.1).

Between 7 and 14 weeks after eclosion, the same individuals from the medium term trial were exposed to a "short term" trial that took place within one day. Five death feigning attempts were performed on each individual during one day. Each attempt was separated by a minute from the time that the individual stopped death feigning or, in the case that the individual did not death feign, from the time of the last tap. Taps were given until the individual feigned death or until 5 taps had been given (Fig.2).

\section{Statistical analysis}

Statistical analyses were performed using a combination of SPSS 17.0, R 2.12.0 and Microsoft Excel 2010. The change in the probability of death feigning over attempts/days was examined using a binary logistic regression. The relationship between death feigning duration and attempt/days was examined by fitting a rank transformed generalized linear mixed effects model. 


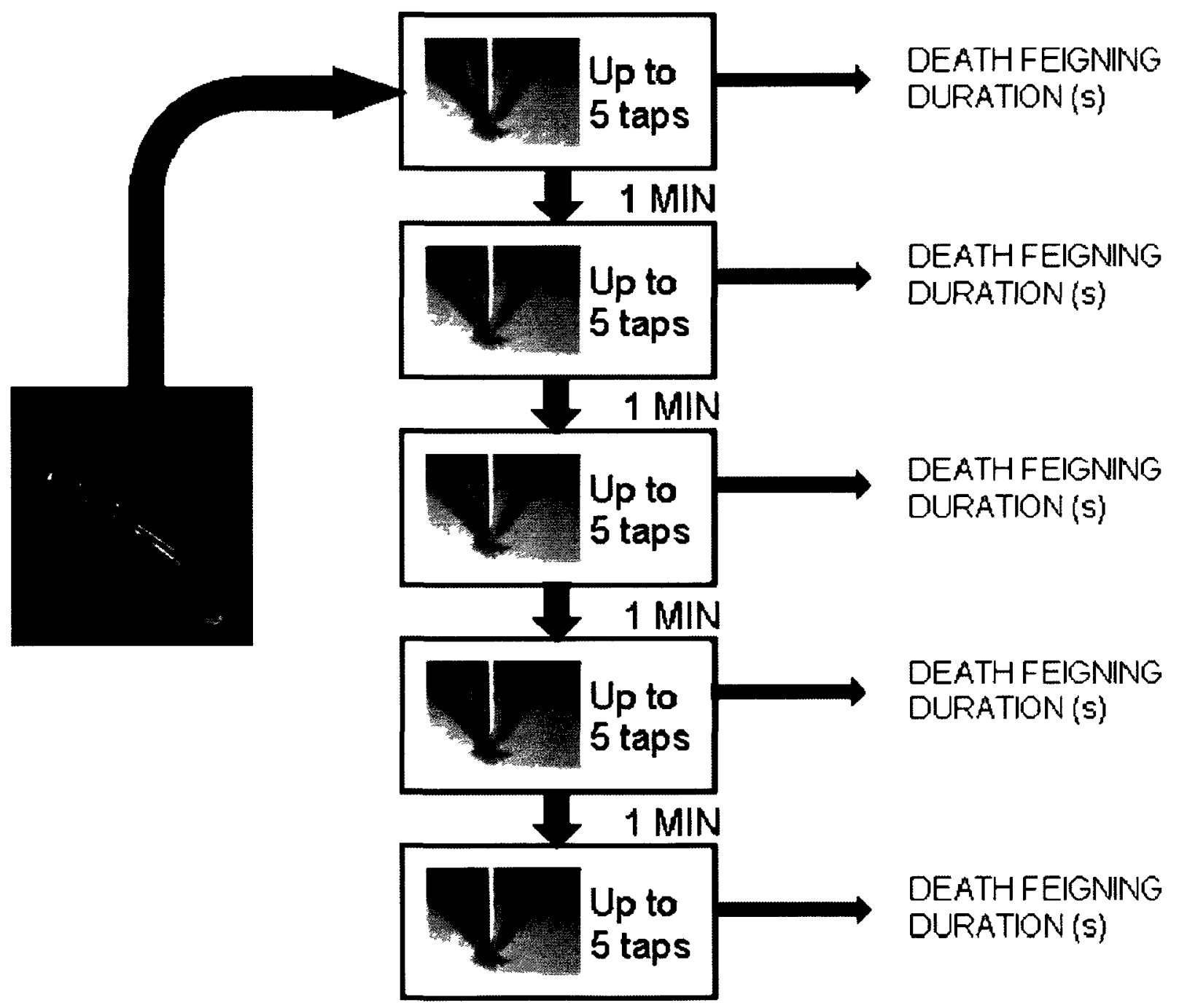

Figure 1. Illustration of the short term death feigning testing. 


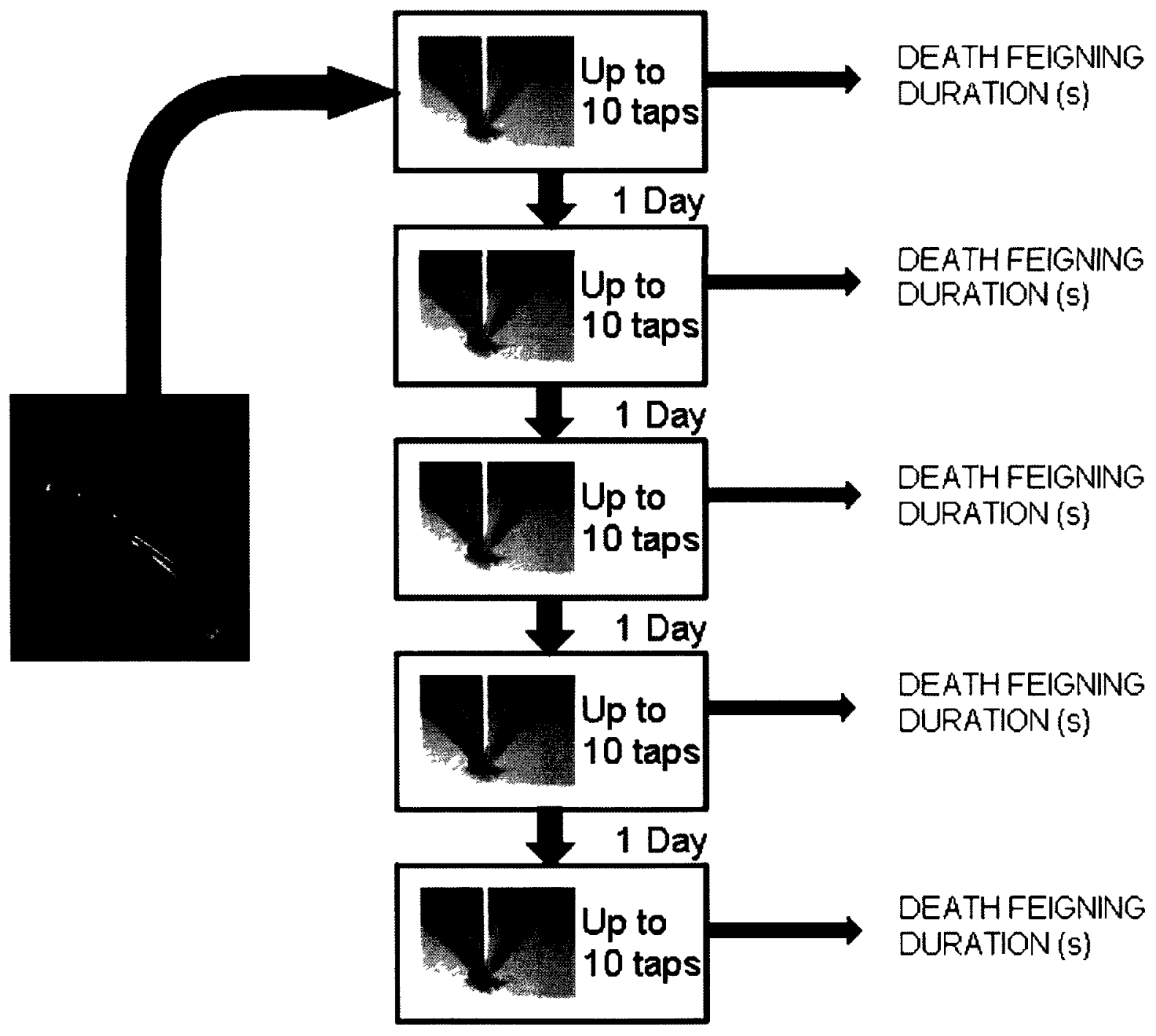

Figure 2. Illustration of the medium term death feigning testing. 
The relationships between average death feigning duration and the probability of death feigning, as well as the relationship between average death feigning duration and the average number of taps, for the short and medium term trials were examined using a univariate GLM. The average death feigning duration and the average number of taps were both taken using only those attempts/days where individuals death feigned ( 6 of out 275 individuals were excluded in the short trials; all 302 individuals were included in the medium trials). Average death feigning duration was also double square root transformed and LN transformed for the short term trials and medium term trials, respectively, to meet the requirements of parametric testing. In a separate test on death feigning propensity, the probability of death feigning was taken as the number of death feigning attempts/days out of 5 attempts/days (with attempts treated as a factor rather than a covariate to allow for non-linearity). Additional factors included in the models above were population, individual age (in weeks), and sex. Individual was included as a random factor in the binary logistic regression and the generalized linear mixed effects model, however, due to the methods used in $\mathrm{R}$ to run the binary logistic regression, I was unable to provide any test statistic or significance testing for the random factor (see below). Although it is important to control for individual variation, it was not the focus of our experiment. The test statistic provided for binary logistic regression in $\mathrm{R}$ is the $\mathrm{z}$ statistic, which is a square rooted Wald statistic. Also, I was unable to provide degrees of freedom for the fitted generalized linear mixed effects models, as the author of the package used in $\mathrm{R}$ to fit the model felt that it would be too difficult to estimate the denominator degrees of freedom for all such designs (Bates 2006). Spearman's rank correlation tests were performed on average death feigning duration, and probability of death feigning for individuals between the short and medium term trials to see if individuals were consistent between trials. 
Repeatability of death feigning duration was measured in the medium term trials using the Intraclass Correlation Coefficient (ICC; Two-way mixed model, absolute agreement between days, single measure; Appendix), which was calculated using SPSS 17.0. ICC (or repeatability) values are generally interpreted as: $<0.4$ is poorly repeatable; 0.4 to 0.75 indicates fair to good repeatability; and $>0.75$ indicates good to excellent repeatability (Rouse et al. 2004). In the short term trials, ICC was not a realistic measure of repeatability due to a trend of increasing death feigning duration over attempts. Therefore, the correlations between subsequent attempts were examined using a Spearman's rank correlation test, to see if the increasing duration trend was consistent between individuals. 


\section{$\underline{\text { Results }}$}

\section{Probability of death feigning}

In the short trials I found that the probability of death feigning significantly increased with increasing attempts (Table 1, Fig.3A). There were no significant differences in death feigning probabilities between the different populations, sexes or age groups (Table 1, Fig.3A). In the medium trials, I likewise found that the probability of death feigning significantly increased with increasing days (Table 1, Fig.3B). There were no significant differences in death feigning probabilities between sexes, populations or age groups (Table 1, Fig.3B). 
Table 1. Binomial logistic regression statistics, and the relationships between the probability of death feigning and several factors for the short and medium trials.

\begin{tabular}{|c|c|c|c|c|}
\hline \multirow{3}{*}{ Trial } & Factor & $z$ & Std. Error & $P$ \\
\hline \multirow{4}{*}{ Short } & Population & 0.667 & 0.803 & 0.505 \\
\cline { 2 - 5 } & Sex & -0.427 & 1.351 & 0.670 \\
\cline { 2 - 5 } & Age & 0.334 & 0.288 & 0.739 \\
\cline { 2 - 5 } & Attempt & 8.021 & 0.151 & $<0.001$ \\
\hline \multirow{3}{*}{ Medium } & Population & 0.467 & 0.353 & 0.640 \\
\cline { 2 - 5 } & Sex & -0.917 & 0.469 & 0.359 \\
\cline { 2 - 5 } & Age & -0.809 & 0.548 & 0.418 \\
\cline { 2 - 5 } & Day & 3.766 & 0.106 & $<0.001$ \\
\cline { 2 - 5 } & & & & \\
\hline
\end{tabular}



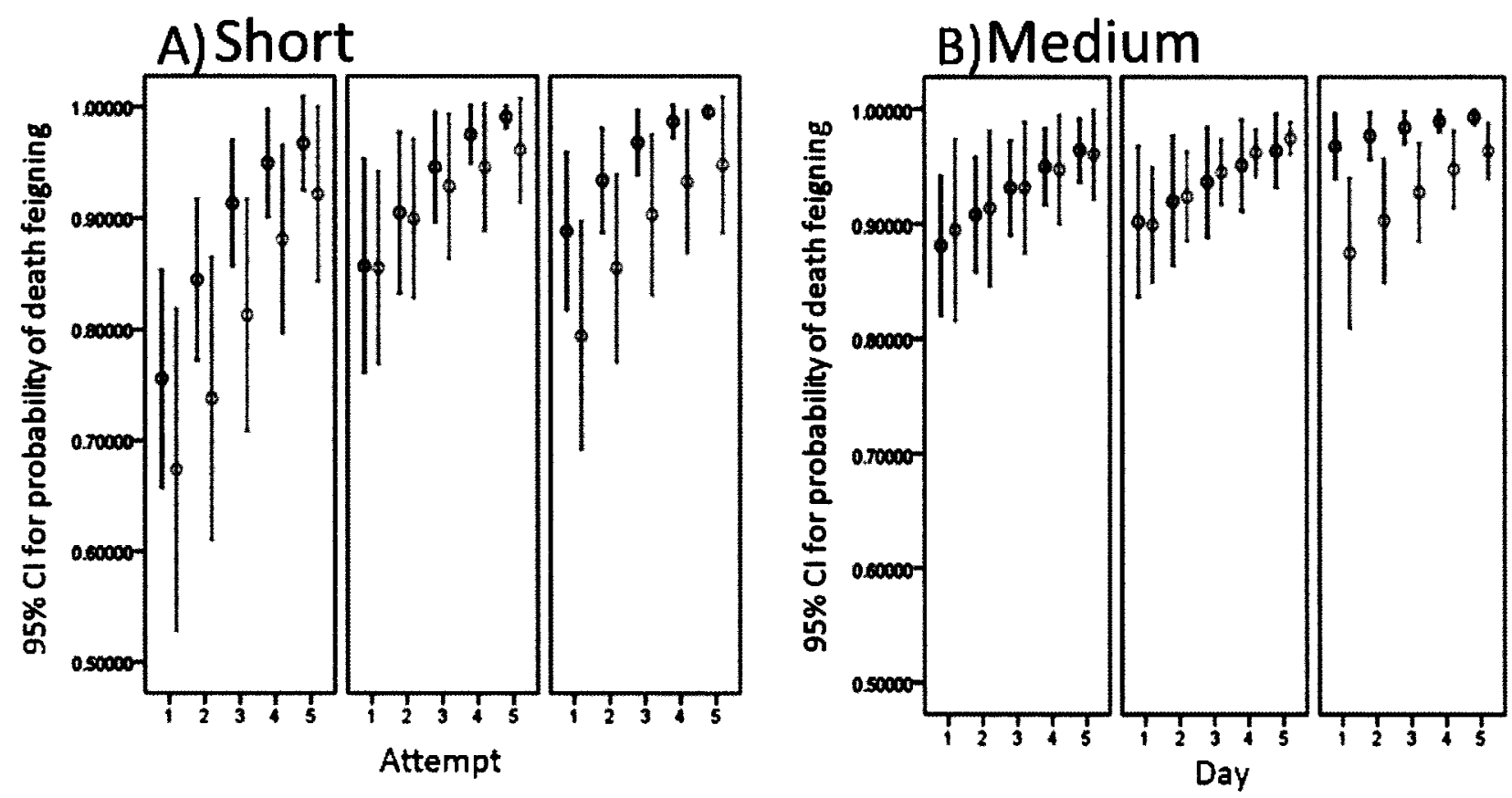

Figure 3. The probability of death feigning versus A) the number of attempts for the short term trials and $\mathrm{B}$ ) the number of days for the medium term trials (error bars are $95 \%$ confidence intervals). From left to right: $\mathrm{C}++, \mathrm{BB}, \mathrm{Croatia}$. The blue circles are females and the green circles are males. 


\section{Death feigning duration}

In the short trials I found that death feigning duration increased significantly over increased attempts (Table 2, Fig.4A). There was also a significant difference in average death feigning duration between sexes, populations, age groups, and individuals (Table 2). Females had a higher duration than males in all populations (Fig. 5), however, only C++ (Table 3) and Croatia (Table 3) females had significantly greater death feigning durations than males. BB had greater death feigning durations, followed by Croatia, and $\mathrm{C}++$ (Fig. 6). The age group results were potentially confounded because populations were tested at different ages. To tease apart the difference between populations and ages, I examined the relationship between death feigning duration and increasing age in each population. I found that death feigning duration did not significantly increase with age within any of the populations (Table 3, Fig.7).

In the medium trials I found that death feigning duration increased significantly over days (Table 2, Fig.4B). There was also a significant difference in death feigning duration between populations, and individuals (Table 2). There was no significant difference between the sexes or the age groups (Table 2). Croatia did not demonstrate a significant positive relationship between death feigning duration and days (Table 3, Fig.4B), while $\mathrm{C}++$ demonstrated a slight but significant positive relationship between death feigning duration and days (Table 3, Fig.4B), and BB demonstrated a strongly significant positive relationship between death feigning duration and days (Table 3, Fig.4B). BB had the highest durations, followed by Croatia and C++ (Fig.8). 
Table 2. Repeated measures generalized linear mixed effect model statistics, and the relationships between death feigning duration and several factors for the short and medium trials. Restricted likelihood ratio tests (RLRT) were used to calculate if there was a significant difference in death feigning durations between individuals.

\begin{tabular}{|l|l|l|l|l|}
\hline Trial & Factors & $\mathrm{t}$ & Std. Error & $\mathrm{P}$ \\
\hline Short & Pop & 2.886 & 18.887 & 0.004 \\
\cline { 2 - 5 } & Sex & -2.752 & 31.196 & 0.006 \\
\cline { 2 - 5 } & Age & 4.837 & 6.178 & $<0.001$ \\
\cline { 2 - 5 } & Attempt & 31.578 & 4.462 & $<0.001$ \\
\cline { 2 - 5 } & Individual & $459.694^{*}$ & NA & $<0.001$ \\
\hline \multirow{5}{*}{ Medium } & Pop & 2.369 & 24.739 & 0.018 \\
\cline { 2 - 5 } & Sex & -1.055 & 34.224 & 0.289 \\
\cline { 2 - 5 } & Age & -0.330 & 39.431 & 0.739 \\
\cline { 2 - 5 } & Day & 3.465 & 6.422 & 0.001 \\
\cline { 2 - 5 } & Individual & $234.451^{*}$ & NA & $<0.001$ \\
\hline
\end{tabular}

* calculated using RLRT. 

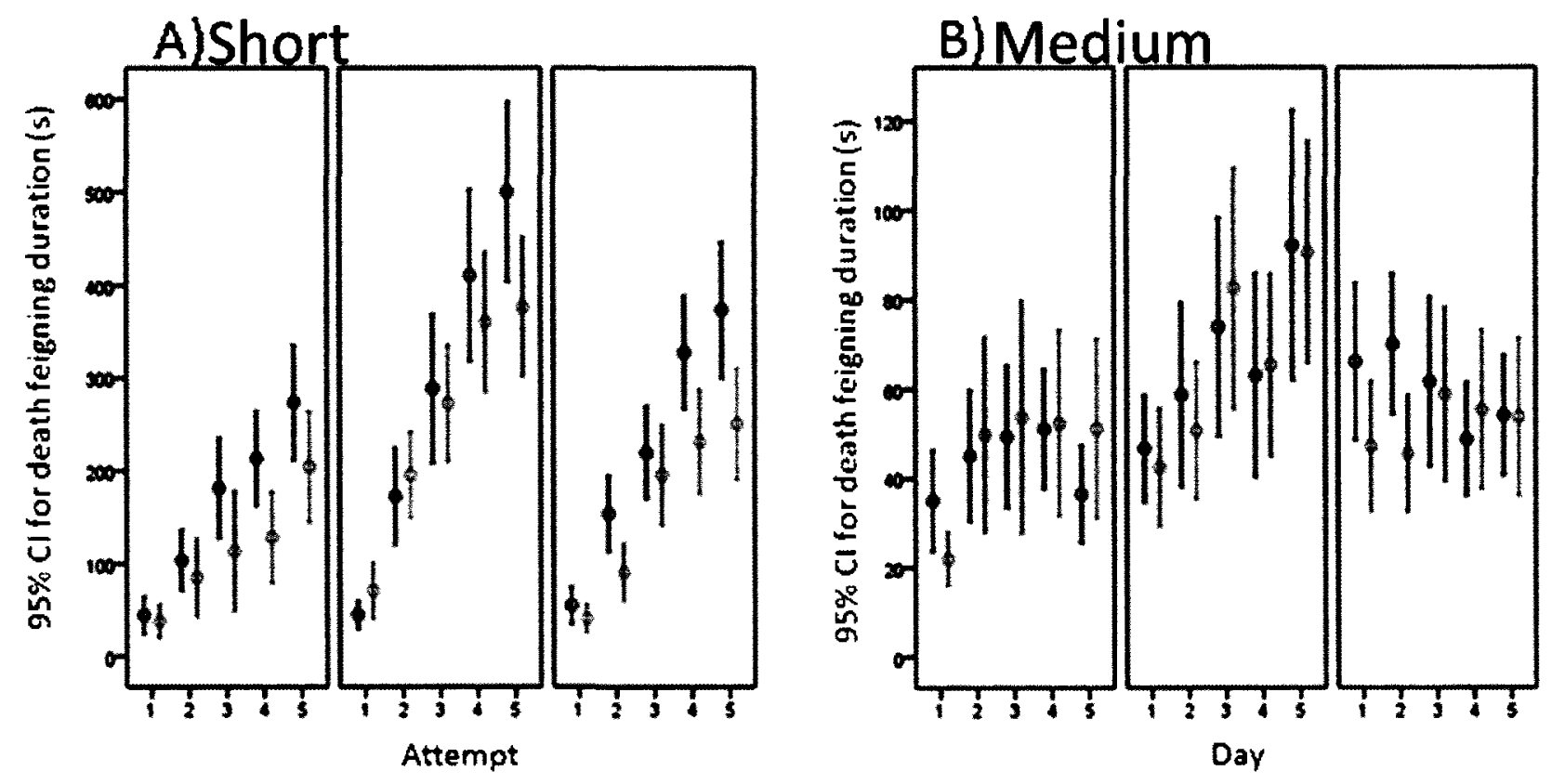

Figure 4. The duration of death feigning (in seconds) versus A) the number of attempts for the short term trials and $\mathrm{B}$ ) the number of days for the medium term trials (with $95 \%$ confidence intervals). From left to right: $\mathrm{C}++, \mathrm{BB}$, Croatia. The blue circles are females and the green circles are males. 


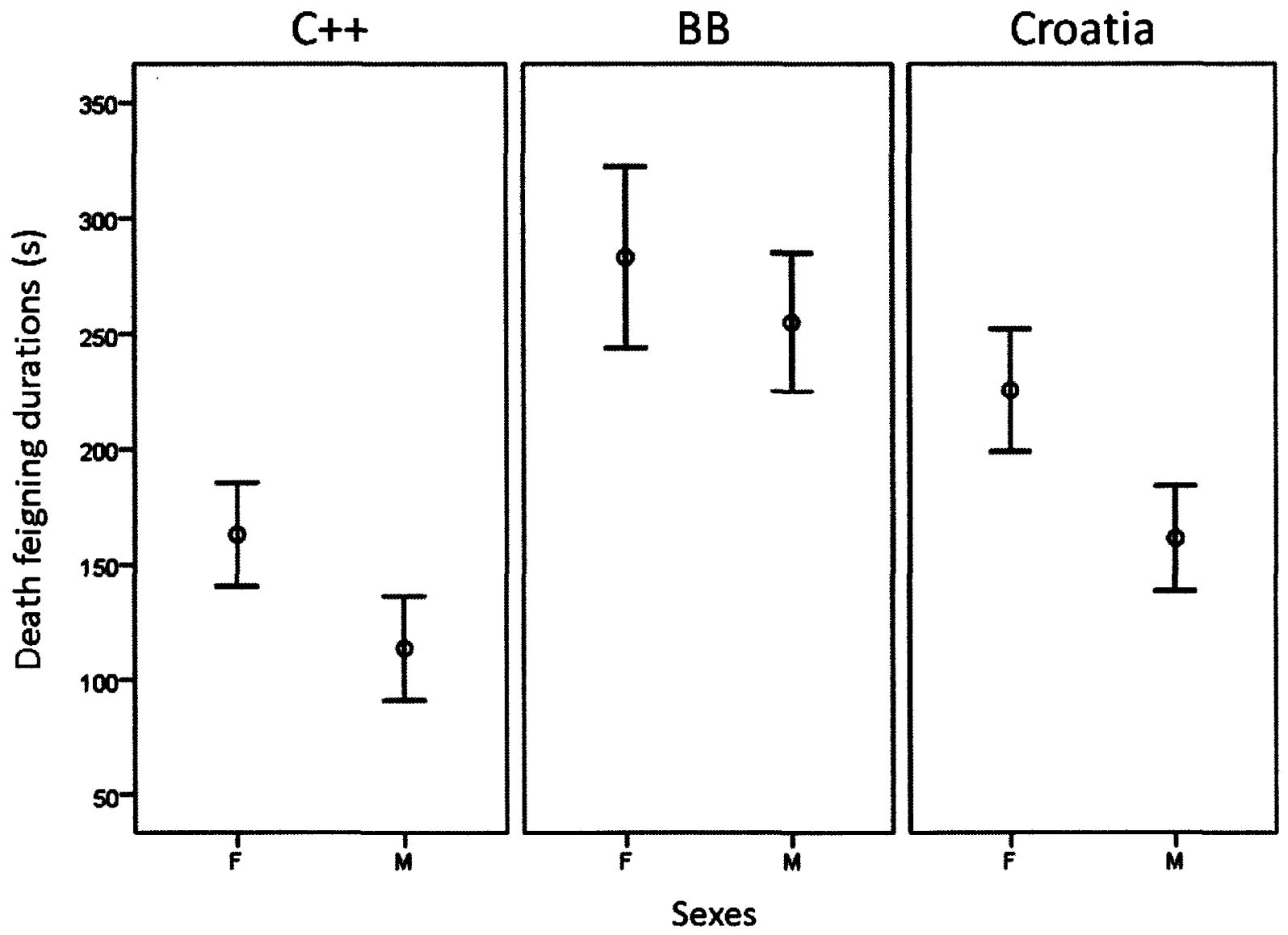

Figure 5. Differences in death feigning durations between the sexes for the different populations in the short trials. 
Table 3. Relationships between death feigning duration and three factors within populations for the short and medium trials.

\begin{tabular}{|c|c|c|c|c|c|}
\hline Trial & Population & Factors & $\mathrm{t}$ & Std. Error & $\mathbf{P}$ \\
\hline \multirow[t]{9}{*}{ Short } & \multirow[t]{3}{*}{$\mathrm{C}++$} & Attempt & 16.071 & 7.405 & 0.000 \\
\hline & & Sex & -2.234 & 56.663 & 0.025 \\
\hline & & Age & -0.271 & 56.912 & 0.783 \\
\hline & \multirow[t]{3}{*}{ BB } & Attempt & 19.522 & 8.121 & 0.000 \\
\hline & & Sex & -0.239 & 58.794 & 0.808 \\
\hline & & Age & 0.343 & 31.245 & 0.727 \\
\hline & \multirow[t]{3}{*}{ Cro } & Attempt & 19.203 & 7.511 & 0.000 \\
\hline & & Sex & -2.513 & 47.967 & 0.012 \\
\hline & & Age & 0.570 & 37.216 & 0.563 \\
\hline \multirow[t]{9}{*}{ Medium } & \multirow[t]{3}{*}{$\mathrm{C}++$} & Day & 2.605 & 11.874 & 0.009 \\
\hline & & Sex & -0.467 & 58.172 & 0.635 \\
\hline & & Age & -3.090 & 58.415 & 0.002 \\
\hline & \multirow[t]{3}{*}{ BB } & Day & 4.635 & 10.402 & 0.000 \\
\hline & & Sex & -0.677 & 62.546 & 0.493 \\
\hline & & Age & 0.599 & 90.584 & 0.544 \\
\hline & \multirow[t]{3}{*}{ Cro } & Day & -1.177 & 11.022 & 0.239 \\
\hline & & Sex & -1.290 & 53.978 & 0.194 \\
\hline & & Age & NA & NA & NA \\
\hline
\end{tabular}




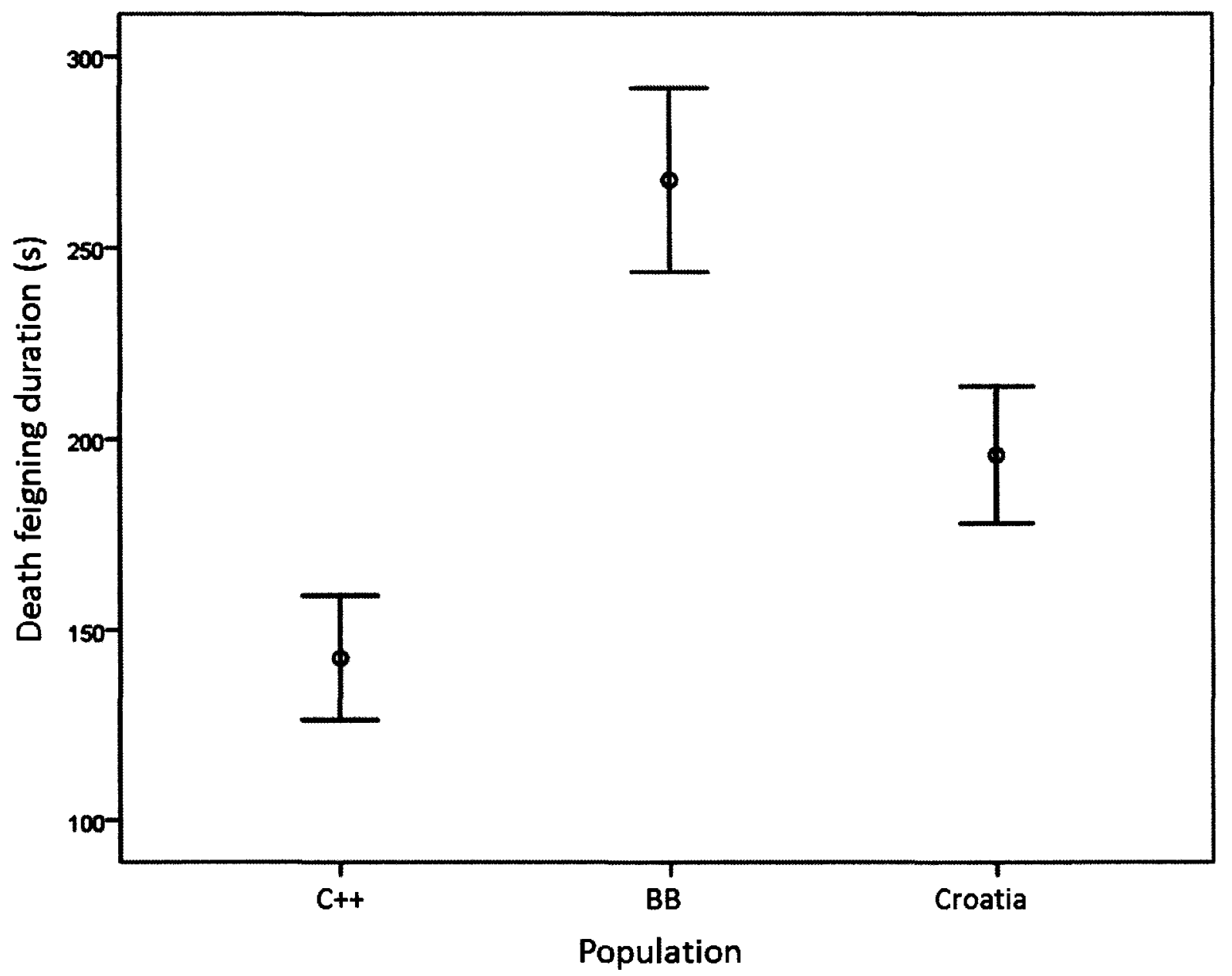

Figure 6. Differences in death feigning durations (in seconds) between populations in the short trials. 


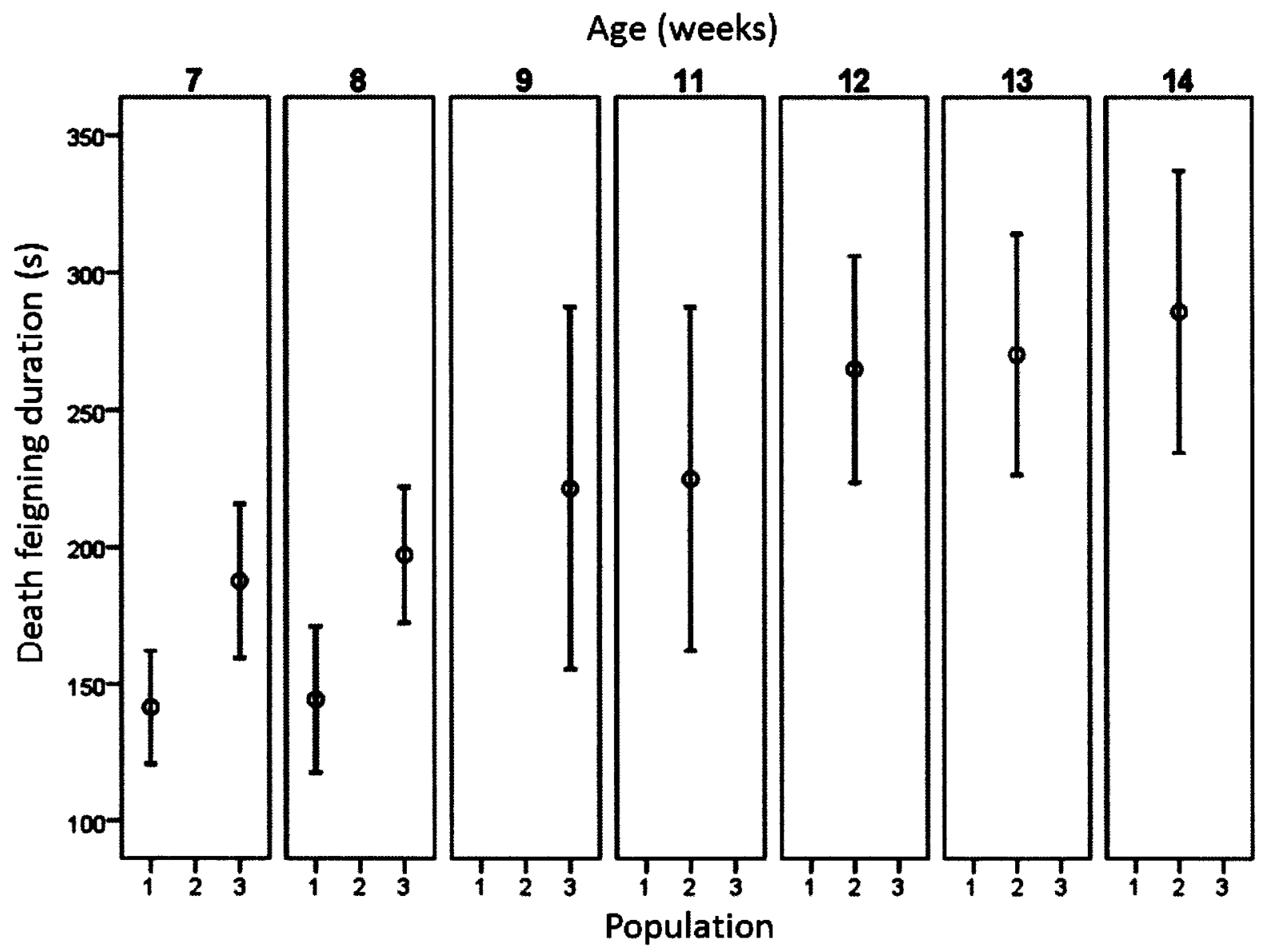

Figure 7. Differences in death feigning durations (in seconds) between populations for each age group in the short trials. From left to right: age groups (in weeks) 7-14. Within each age group, 1-3 are $\mathrm{C}++, \mathrm{BB}$ and Croatia, respectively. 


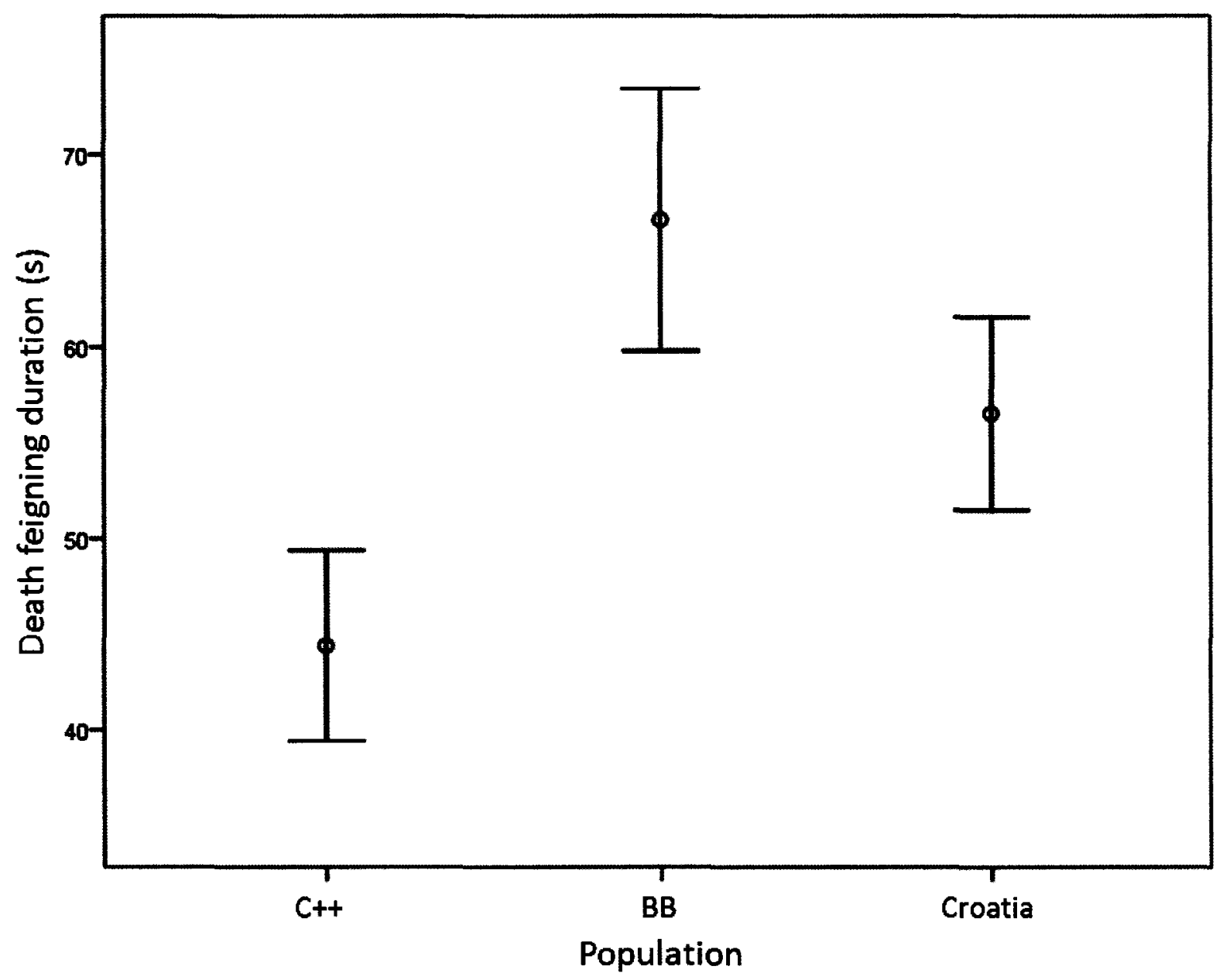

Figure 8. Differences in death feigning durations (in seconds) between the populations for the medium trial. 


\section{Average death feigning duration and probability of death feigning}

In the short trials I found a significant positive relationship between average death feigning duration and the probability of an individual death feigning (Table 4, Fig.9A), indicating that those that death feign more frequently also death feign for longer when they do. There was a significant difference in average death feigning duration between the sexes (Table 4), with females having a higher average death feigning duration than males (Fig.10). There was no significant difference in average death feigning duration between the populations or age groups (Table 4).

In the medium trials I found a significant positive relationship between average death feigning duration and the probability of an individual death feigning (Table 4, Fig.9B), indicating that those individuals that death feign more, death feign for longer. There was a significant difference in average death feigning duration between populations (Table 4), with BB having the greatest average duration, followed by Croatia and $\mathrm{C}++$ (Fig.11). There was no significant difference in average death feigning duration between the sexes, or different ages (Table 4).

\section{Average death feigning duration and average number of taps}

In the short trials I found a significant negative relationship between average death feigning duration and the average number of taps required to induce death feigning (Table 4, Fig. 12A). In the medium trials I found a significant negative relationship between average death feigning duration and the average number of taps required to induce death feigning (Table 4 , Fig.12B). This indicates that in both trials, those individuals that death feign longer require less taps to induce death feigning. The other factors in the model (sex, age, population) are the same as the previous section on average death feigning duration and probability (Table 4). 
Table 4. Relationship between average death feigning duration and several factors in the short and medium trials.

\begin{tabular}{|c|c|c|c|c|c|}
\hline Trial & Factor & $F$ & Mean Square & $\mathrm{df}$ & $P$ \\
\hline \multirow[t]{5}{*}{ Short } & Sex & 5.166 & 1.691 & 1,268 & 0.024 \\
\hline & Population & 2.869 & 0.939 & 2,268 & 0.092 \\
\hline & Age & 0.437 & 0.143 & 5,268 & 0.822 \\
\hline & $\begin{array}{c}\text { Average } \\
\text { number of taps }\end{array}$ & 34.041 & 11.142 & 1,268 & $<0.001$ \\
\hline & $\begin{array}{l}\text { Prob. of death } \\
\text { feign }\end{array}$ & 10.687 & 3.498 & 1,268 & 0.001 \\
\hline \multirow[t]{5}{*}{ Medium } & Sex & 0.296 & 0.101 & 1,301 & 0.587 \\
\hline & Population & 13.500 & 4.589 & 2,301 & $<0.001$ \\
\hline & Age & 2.940 & 0.999 & 1,301 & 0.087 \\
\hline & $\begin{array}{c}\text { Average } \\
\text { number of taps }\end{array}$ & 59.789 & 20.323 & 1,301 & $<0.001$ \\
\hline & $\begin{array}{l}\text { Prob. of death } \\
\text { feign }\end{array}$ & 8.040 & 2.733 & 1,301 & 0.005 \\
\hline
\end{tabular}



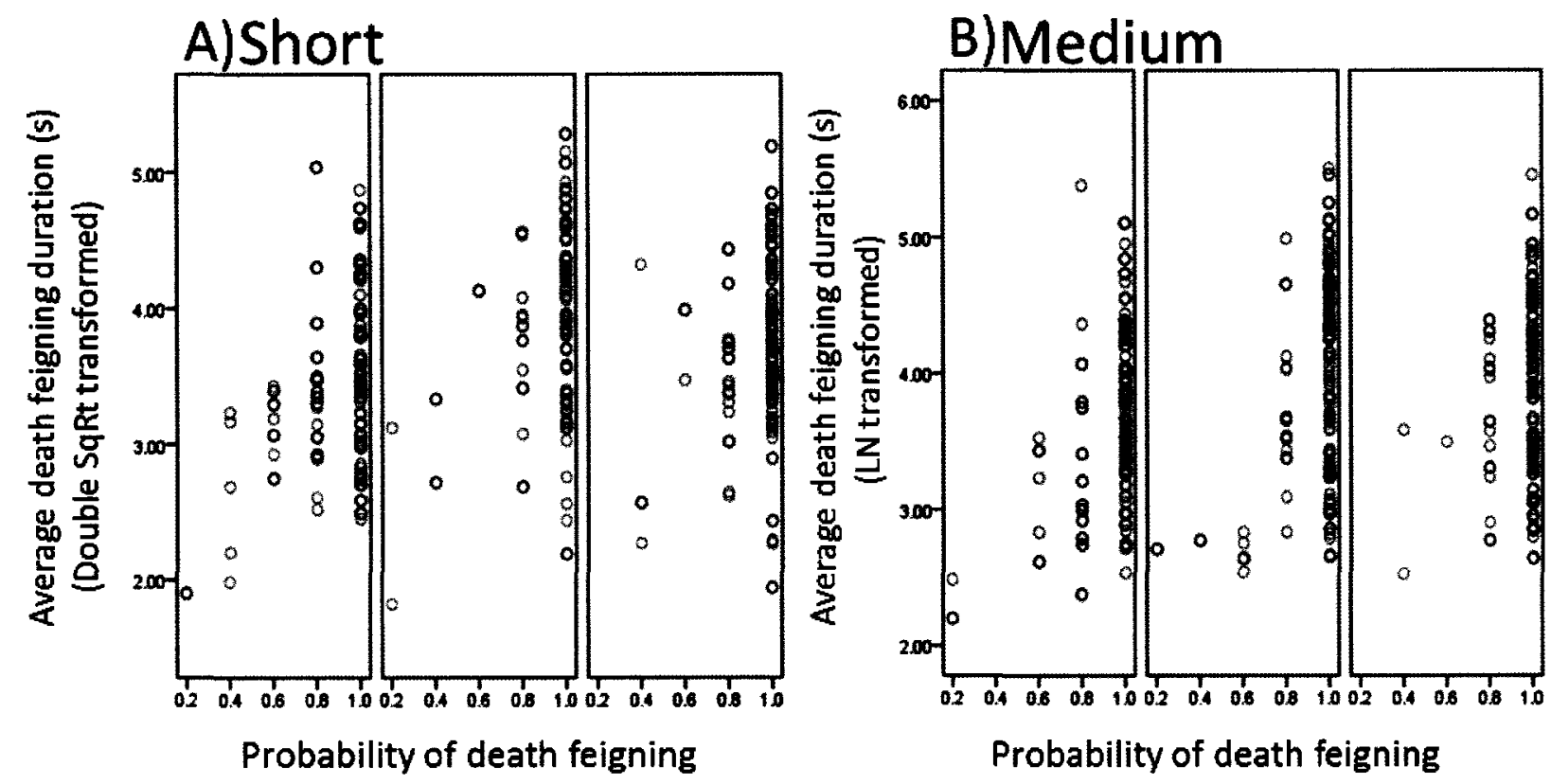

Figure 9. The average duration of death feigning (in seconds) versus A) the probability of death feigning for the short term trials and $\mathrm{B}$ ) the probability of death feigning for the medium term trials. From left to right: $\mathrm{C}++, \mathrm{BB}$, Croatia. The blue circles are females and the green circles are males. 


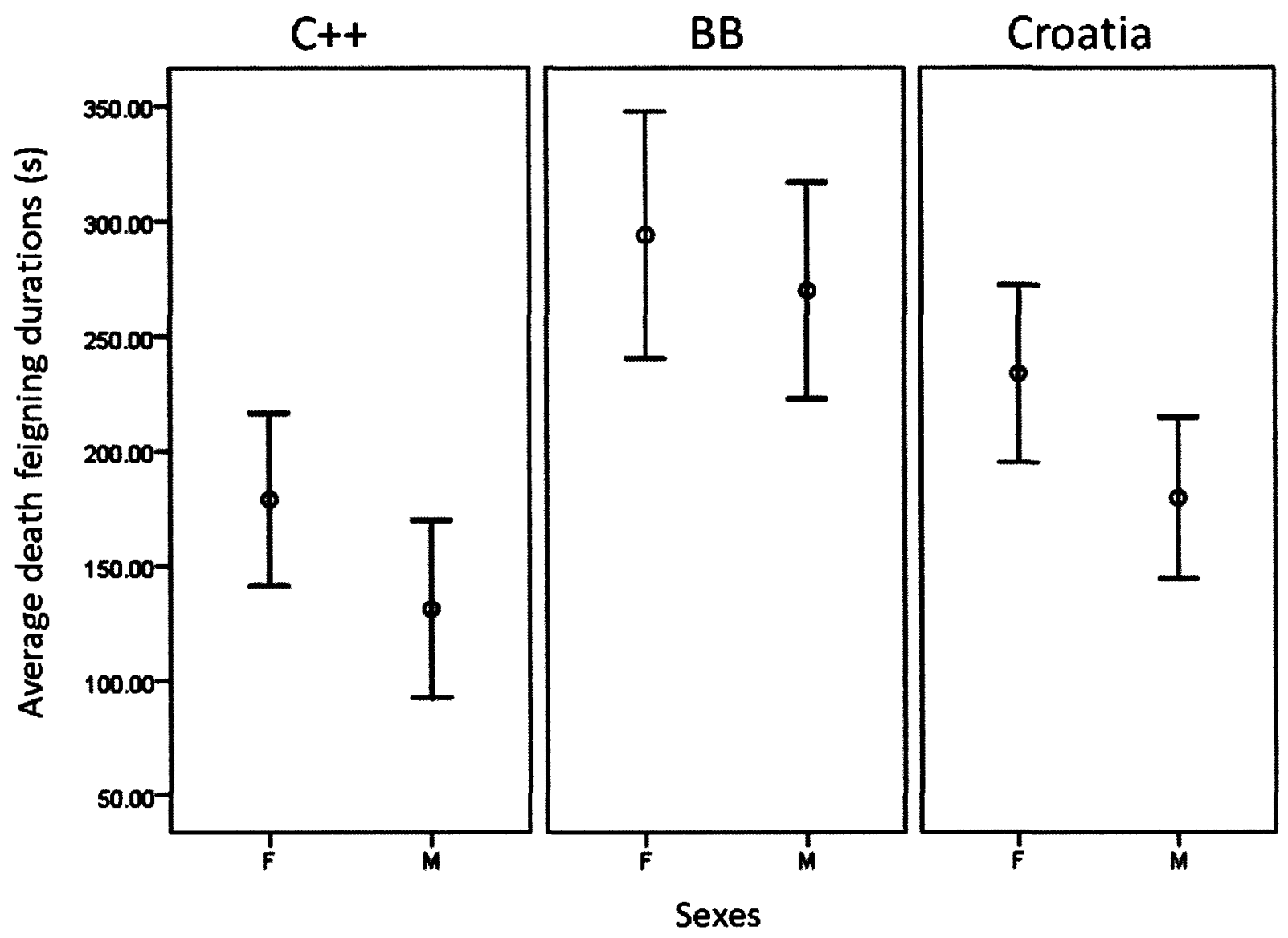

Figure 10. Average death feigning durations (in seconds) for the sexes for the different populations in the short trials. 


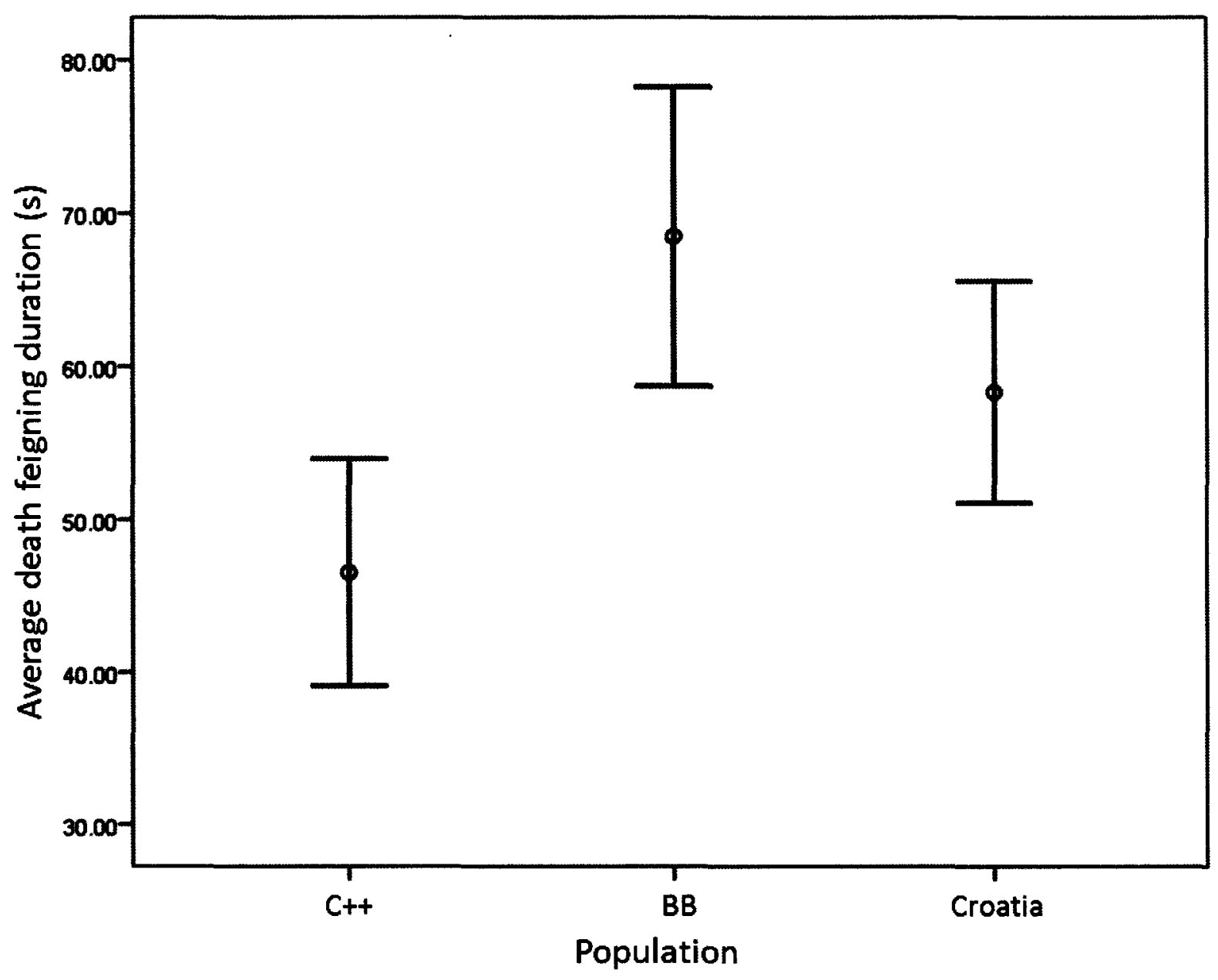

Figure 11. Average death feigning duration (in seconds) for the populations in the medium trials. 

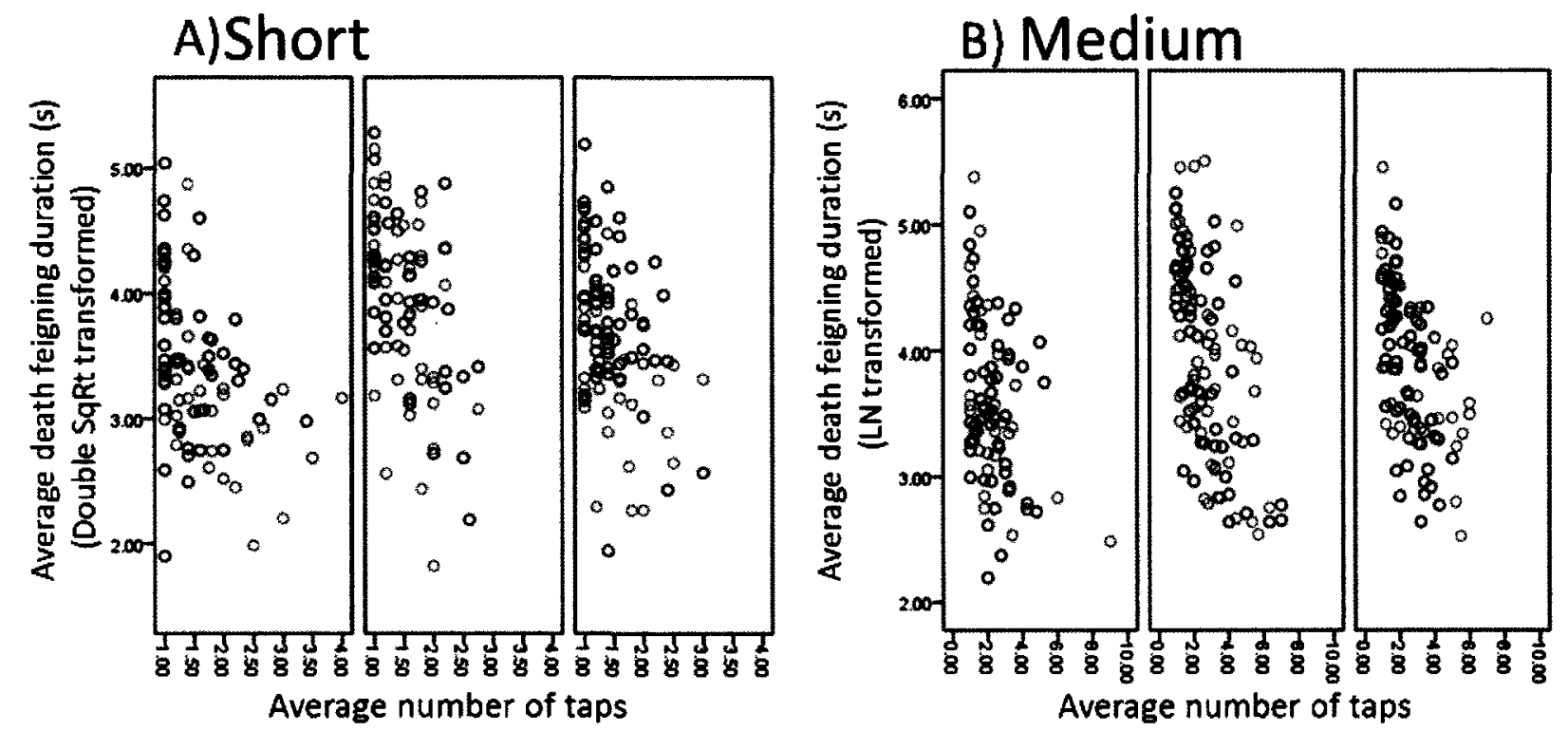

Figure 12. The average duration of death feigning (in seconds) versus A) the average number of taps required to induce death feigning in the short term trials and $\mathrm{B}$ ) the average number of taps required to induce death feigning in the medium term trials. From left to right: $\mathrm{C}++, \mathrm{BB}$, Croatia. The blue circles are females and the green circles are males. 


\section{Correlations between short and medium trials}

There was a significant positive correlation between average death feigning durations for individuals between short and medium term trials $(\rho=0.384, \mathrm{~N}=267, \mathrm{P}<0.001$; Fig. $13 \mathrm{~A})$, indicating that high death feigning duration and low death feigning duration individuals were consistent between trials.

There was also a significant positive correlation between probabilities of death feigning for individuals between short and medium term trials $(\rho=0.370, N=2737, P<0.001$; Fig.13B), indicating that individuals who death feigned more or death feigned less were consistent in the amount of times they death feigned between trials.

\section{Repeatability of death feigning duration}

In the short trials, with the exception of the $\mathrm{C}++$ females between attempt 1 and 2 , all the populations and sexes showed significant correlations between subsequent attempts (Table 5), indicating that the previously discussed pattern of increasing death feigning duration over attempt was consistent between individuals.

In the medium trials I found a range of ICC values from 0.243 in $\mathrm{C}++$ males $(\mathrm{ICC}=0.243$, $95 \% \mathrm{CI}=0.110-0.417$, Table 6 ) to 0.465 in $\mathrm{BB}$ females ( $\mathrm{ICC}=0.465,95 \% \mathrm{CI}=0.335-0.605$, Table 6). Since all these values are all below 0.4 , with the exception of BB females, it shows a poor repeatability for death feigning duration, indicating a high amount of variability in death feigning duration between days. 

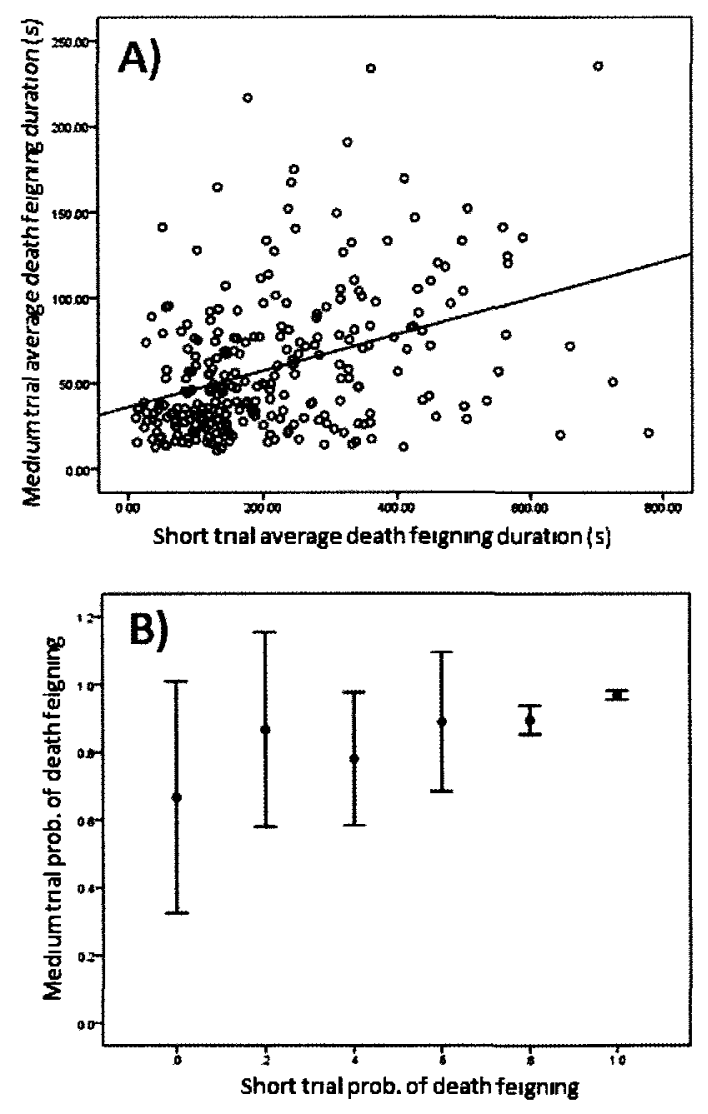

Figure 13. Spearman's rank correlations between individuals in the short trials and the medium trials. A) Correlations between individuals for average death feigning durations (s); B) Correlations between individuals for probabilities of death feigning. 
Table 5. Spearman's rank correlations testing the correlation in death feigning duration values between subsequent attempts in the short term trial. Tests were done for each sex in each population.

\begin{tabular}{|c|c|c|c|c|c|c|}
\hline Pop & Sex & Stat & $\begin{array}{c}1-2 \\
\text { Attempt }\end{array}$ & $\begin{array}{c}2-3 \\
\text { Attempt }\end{array}$ & $\begin{array}{c}3-4 \\
\text { Attempt }\end{array}$ & $\begin{array}{c}4-5 \\
\text { Attempt }\end{array}$ \\
\hline \multirow[t]{4}{*}{$\mathrm{C}++$} & \multirow{2}{*}{$\begin{array}{c}\text { Male } \\
(\mathrm{N}=36)\end{array}$} & $\rho$ & 0.666 & 0.670 & 0.690 & 0.476 \\
\hline & & P-value & 0.000 & 0.000 & 0.000 & 0.003 \\
\hline & \multirow{2}{*}{$\begin{array}{l}\text { Female } \\
(\mathrm{N}=51)\end{array}$} & $\rho$ & 0.250 & 0.716 & 0.600 & 0.639 \\
\hline & & P-value & 0.077 & 0.000 & 0.000 & 0.000 \\
\hline \multirow[t]{4}{*}{ BB } & \multirow{2}{*}{$\begin{array}{c}\text { Male } \\
(\mathrm{N}=49)\end{array}$} & $\rho$ & 0.314 & 0.675 & 0.690 & 0.846 \\
\hline & & P-value & 0.028 & 0.000 & 0.000 & 0.000 \\
\hline & \multirow{2}{*}{$\begin{array}{l}\text { Female } \\
(\mathrm{N}=40)\end{array}$} & $\rho$ & 0.616 & 0.761 & 0.706 & 0.620 \\
\hline & & P-value & 0.000 & 0.000 & 0.000 & 0.000 \\
\hline \multirow[t]{4}{*}{ Cro } & \multirow{2}{*}{$\begin{array}{c}\text { Male } \\
(\mathrm{N}=46)\end{array}$} & $\rho$ & 0.589 & 0.693 & 0.777 & 0.598 \\
\hline & & P-value & 0.000 & 0.000 & 0.000 & 0.000 \\
\hline & \multirow{2}{*}{$\begin{array}{l}\text { Female } \\
(\mathrm{N}=53)\end{array}$} & $\rho$ & 0.560 & 0.461 & 0.553 & 0.702 \\
\hline & & P-value & 0.000 & 0.001 & 0.000 & 0.000 \\
\hline
\end{tabular}


Table 6. Intraclass correlation coefficients for each sex in each population for the medium term trial.

\begin{tabular}{|c|c|c|c|c|c|}
\hline Trial & Pop & Sex & ICC & CI & df \\
\hline Medium & C++ & Male & 0.243 & $0.110-$ & 36,144 \\
& & & & 0.417 & \\
\cline { 3 - 6 } & & Female & 0.352 & $0.228-$ & 51,204 \\
& & & & 0.496 & \\
\cline { 3 - 6 } & BB & Male & 0.356 & $0.241-$ & 60,240 \\
& & & 0.487 & \\
\cline { 3 - 6 } & & Female & 0.465 & $0.335-$ & 48,192 \\
& & & & 0.605 & \\
\cline { 3 - 6 } & \multirow{2}{*}{ Cro } & Male & 0.273 & $0.148-$ & 46,184 \\
& & & & 0.427 & \\
\cline { 3 - 6 } & & Female & 0.290 & $0.176-$ & 55,220 \\
& & & 0.428 & \\
\hline
\end{tabular}




\section{$\underline{\text { Discussion }}$}

It is important to know how repeatable and variable death feigning behaviour is within a species because, knowing whether an individual is a death feigner or not, or even if that classification is appropriate, will allow for further experiments to assess the relationship between death feigning and other life history traits, as well as allowing for ultimate hypothesis testing. For my thesis, I quantified the variability and repeatability of death feigning in $T$. castaneum. Such research is a necessary precursor to my subsequent investigation of the relationship between death feigning and chemical defence (chapter 3).

The probability of an individual death feigning increased with successive attempts, both within a day (short trials) and over multiple days (medium trials). This shows that a high proportion of individuals in my populations death feigned with enough provocation, and that individuals in previous studies who were classified as non-death feigners may have been death feigners had they been provoked more.

The duration of death feigning increased over successive attempts in the short trials for all the populations. While all the populations showed similar trends of increasing death feigning duration, there were differences between populations in terms of the size of death feigning durations (a result that was also evident in the medium trials), indicating that similar rearing conditions over a large number of generations was not enough to fully remove differences between the populations. Additionally, I found a difference between the sexes, which is contrary to previous studies examining death feigning in T. castaneum (Prohammer and Wade 1981; Miyatake et al. 2004). The results in the medium trials were not as consistent across populations, 
with each population displaying different responses to successive attempts to induce death feigning, however, none of the populations exhibited negative trends.

The results for probability, and death feigning duration show that the individuals are not habituating to the attempts to induce death feigning, which is contrary to some previous studies in other species (Jones 1977; Watsky and Gruber 1990). This would indicate that individuals retained some previous memory of the threat, and increased their anti-predator response accordingly. As a result, individuals who did not death feign at first were likely to death feign given enough provocation, and often increased the amount they death feigned given increased provocation. I cannot be sure how long this increasing trend would continue, as there is previous evidence in other species for variability in how long individuals continue to death feign after successive attempts to induce death feigning (Francq 1969). I imagine that increasing the number of attempts to induce death feigning would eventually show habituation to the threat.

Interestingly, those individuals that death feign more often, and those individuals that require less provocation to death feign will death feign for longer. These results were not entirely surprising. While we cannot classify individuals as death feigners and non-death feigners because there were very few individuals that did not death feign at some point during the trials, we could imagine there are individuals who are "greater" death feigners than others. One could assume that individuals that death feign more or require less provocation to death feign would be "greater" death feigners, and would likely death feign for longer durations.

Individuals displayed a poor repeatability of death feigning duration between days, which was contrary to results from closely related species (Nakayama et al. 2009). Differences in repeatability between species (T. confusum and T. castaneum) and populations (Croatia, BB, 
$\mathrm{C}++)$ could be because heritability is population specific. Death feigning duration, at least taken from a daily measure, would not be a useful indicator of death feigning, as it is highly variable between days. However, individuals during the short trials consistently demonstrated a trend of increasing death feigning duration between successive attempts. Additionally, I found that average death feigning durations, and probabilities of death feigning were consistent in individuals between short and medium trials. These results, combined, suggest that death feigning duration values may fluctuate considerably between days but the average durations of all positive death feigning attempts are consistent. Thus, I have established that average death feigning duration could be used as a measure of how "great" a death feigner an individual is. Taking these results into account, it would appear that taking the average of the death feigning duration results for all the short trial attempts would be an appropriate indicator of death feigning to use for examining the relationship between death feigning and chemical defence, as the medium trials were highly variable, with some individuals showing consistency, while others fluctuated considerably.

In conclusion, I found that $T$. castaneum individuals became more likely to death feign over successive attempts, that they death feigned for longer over successive attempts, and that those individuals who were more likely to death feign or death feigned with less provocation death feigned for longer. I also found that death feigning duration between days is not very repeatable, whereas individuals demonstrated consistent increases in death feigning duration over successive attempts in the short trials. These results have helped to establish that I cannot categorize individuals as death feigners or non-death feigners. A better classification method may be a continuous scale using the average death feigning duration taken from all five death feigning attempts in the short trials. Therefore, I will use the average death feigning duration 
from the short term trial as a measure of death feigning for examining the relationship between death feigning and chemical defence. 


\section{Chapter 3 - The relationship between chemical defence and death feigning in Tribolium castaneum}

\section{Introduction}

Death feigning is a secondary anti-predator defence, wherein the individual enters a state of immobility after contact with a predator (Edmunds 1974). It is a widespread behaviour across a wide range of taxa, however, despite its widespread presence, research into the ultimate causes of death feigning is nascent and ongoing. There are several hypotheses that have been proposed that attempt to explain why death feigning evolved and why it is widespread (Chapter 1). I am interested in examining the proposed hypothesis that death feigning has co-evolved with chemical defence, and that the two anti-predator defences are linked and work together (Miyatake et al. 2004; Ruxton 2006).

The possibility that intra-specific variations in the propensity to death feign may be related to intra-specific variation in chemical defence has long been speculated (Miyatake et al. 2004). In particular, while performing a study comparing the survival rates of death feigning and non-death feigning individuals, Miyatake et al. (2004) presented red flour beetles (Tribolium castaneum) to female Adanson jumping spiders (Hasarius adansoni), and found that the jumping spiders always dropped the beetles after picking them up. Those individuals that death feigned survived, while those that did not were subsequently eaten by the spiders. They concluded that one reason the spiders could be dropping the beetles was because of the beetles' chemical defences, and that the extent of chemical defence may be highly correlated with the propensity to death feign. 
The results of the above study were briefly touched on by Ruxton (2006) in a short review of recent developments in death feigning literature. He proposed that death feigning could act as an honest signal of chemical defence, with immobility indicating to predators that a prey is chemically defended, and as a result, does not have to attempt to escape. This proposal was influenced by evidence from a previous study that showed that some chemically defended butterflies had lower flight speeds, signalling that they had no need to fear capture (Sherratt et al. 2004; Srygley 2004). If this theory were true then it would predict a higher concentration of defensive chemicals in death feigning individuals compared to individuals that were less prepared to death feign.

Miyatake et al. (2009) tested the relationship between chemical defence and death feigning in $T$. castaneum by examining the difference in concentrations of methyl-1,4benzoquinone (MBQ) between male death feigners and non-death feigners. MBQ is a chemical that is noxious to predators (Happ 1968), and forms one of the three primary chemical components that make up the $T$. castaneum defence secretion (Unruh et al. 1998; Villaverde et al. 2007). The authors found no difference between the concentrations of MBQ in death feigners and non-death feigners. This, as well as additional studies they performed that showed increased survivorship of death feigners over non-death feigners when grouped together with a predator, led them to conclude that death feigning and chemical defence are not related, that death feigning is not an honest signal for chemical defence, and that the success of death feigning is reliant on the presence of non-death feigners distracting predators away from death feigners.

While the above study was indicative, it does not entirely rule out the honest signal hypothesis for death feigning. In particular, the study only examined one defensive chemical, 
namely MBQ. There are three primary chemical components in the T. castaneum defence secretion: MBQ, ethyl-1,4-benzoquinone (EBQ), and 1-pentadecene (PENT) (Unruh et al. 1998; Villaverde et al. 2007). PENT has the highest concentration in the defence secretion, followed by EBQ and MBQ (Unruh et al. 1998; Villaverde et al. 2007). EBQ and MBQ are noxious to predators, while there is evidence that PENT repels conspecifics (Unruh et al. 1998; Arnaud et al. 2002; Villaverde et al. 2007). There is also speculation that PENT facilitates the absorption of MBQ and EBQ by predators (Von Endt and Wheeler 1971). While MBQ is not related to death feigning (at least in the former study), it remains possible that EBQ or PENT may be. Therefore an examination of all three chemicals would provide a more comprehensive understanding of the relationship between death feigning and chemical defence.

In addition, Miyatake et al. (2009) did not consider the potential role of sexual differences in chemical defenses, which may potentially obscure any relationship between chemical defence and death feigning. Thus, there is evidence that defensive chemical concentrations differ between sexes in T. castaneum (Unruh et al. 1998), while differences in the death feigning frequency and duration between sexes have been reported in at least some beetle species (Callosobruchus maculatus and Callosobruchus chinensis) (Miyatake et al. 2008). While there is no evidence of sexual differences in death feigning behaviour in Tribolium species in the literature (Prohammer and Wade 1981; Nakayama et al. 2009), I did see a difference between sexes in death feigning durations (Chapter 2). Therefore, if death feigning is related to chemical defence, and there is a difference in death feigning behaviour between sexes, perhaps we will also see a difference in chemical concentrations between sexes. 
Based on prior research I performed (Chapter 2), I was unable to use the death feigner/ non-death feigner classification scheme that Miyatake et al. $(2004,2009)$ used because it was more continuous and not a binary variable. As a result, in this study I investigated the relationship between chemical defence and average death feigning duration (as well as the propensity to death feign), testing all three chemical components of the $T$. castaneum defensive secretion. If the honest signal hypothesis was correct, we would expect a positive relationship between average duration (or its propensity) and all the chemical concentrations. I used two different methods to test the hypothesis: 1) An SPME (Solid Phase Micro Extraction) fibre rub that was used to test the amount of chemicals emitted by individuals when provoked, and, later on the same individuals, 2) a hexane rinse that was used to test the total amount of chemicals that could be released, which was performed a day after the SPME rub tests. 


\section{$\underline{\text { Methods }}$}

\section{$\underline{\text { Study species }}$}

Croatia, a population of $T$. castaneum initially taken from a sampling site in Croatia, former Yugoslavia, over ten years ago and supplied by Dr. Charles Goodnight of the University of Vermont, was used for this study. Individuals were maintained in canning jars, in a mixture of $95 \%$ whole wheat flour and $5 \%$ brewer's yeast by weight. Cultures were stored in an incubator at approximately $29^{\circ} \mathrm{C}$.

Experimental design

\section{Selection of Individuals}

Final instar larvae and pupae were selected and separated into $1.5 \mathrm{~mL}$ microtubes, filled with $1.0 \mathrm{~mL}$ of $95 \%$ whole wheat flour and $5 \%$ brewer's yeast by weight. Microtubes were checked every week for adult eclosion. Once adults had eclosed, individuals were sexed by identification of prothoracic femoral setiferous sex patches (males have them, females do not). A total of 60 males and 60 females were selected for the study. These individuals were divided between two separate groups of tests: Group1, composed of 33 males and 18 females, was tested in late September and early October, while Group2, composed of 27 males and 42 females, was tested in January and February.

\section{Duration of death feigning}

Death feigning was induced, under a dissecting microscope, by taping individuals between the prosternum and mesosternum with metal tweezers. Based on findings from the 
previous chapter, I was unable to use a single estimate of death feigning duration to compare with the chemical concentrations because there was too much variation in the death feigning durations. As a result, I used an average death feigning duration that was calculated from 5 separate death feigning attempts, with up to five taps per attempt. Each attempt was separated by a minute from the time that the individual stopped death feigning or, in the case that the individual did not death feign, from the time of the last tap. Taps were given until the individual feigned death or until five taps had been given. Death feigning duration was measured with a stopwatch. Groupl death feigning duration was sampled between 9 and 11 weeks after eclosion, while Group2 death feigning duration was sampled between 7 and 9 weeks after eclosion. Average duration of death feign was calculated only for the taps which induced a death feign (zeros were not counted) (Fig.1).

\section{Chemical concentration}

Chemical concentrations were measured at the Atlantic Forestry Center in Fredericton, NB, with the help of Dr. Gaetan Leclair and Dr. Peter Silk. Chemical concentrations were measured in individuals using two different methods. The first method was an SPME fibre rub, which was used to capture any emitted chemical on the beetle's body. Each individual was picked up with live insect forceps (approximately centered over the area between the prosternum and mesosternum). An SPME fibre (polydimethylsiloxane coated, 100 microns thick, Aldrich, Canada, part 57300-U, coded red), locked into a SPME holder (Supelco, part 57330), was rubbed over the posterior end of the beetle 40 times to ensure that any chemical present would be picked up. The contaminated SPME fibre was inserted into the syringe holder of an Agilent 6890 Gas Chromatograph (GC) coupled to a 5973 mass selective detector using helium as a carrier gas. 


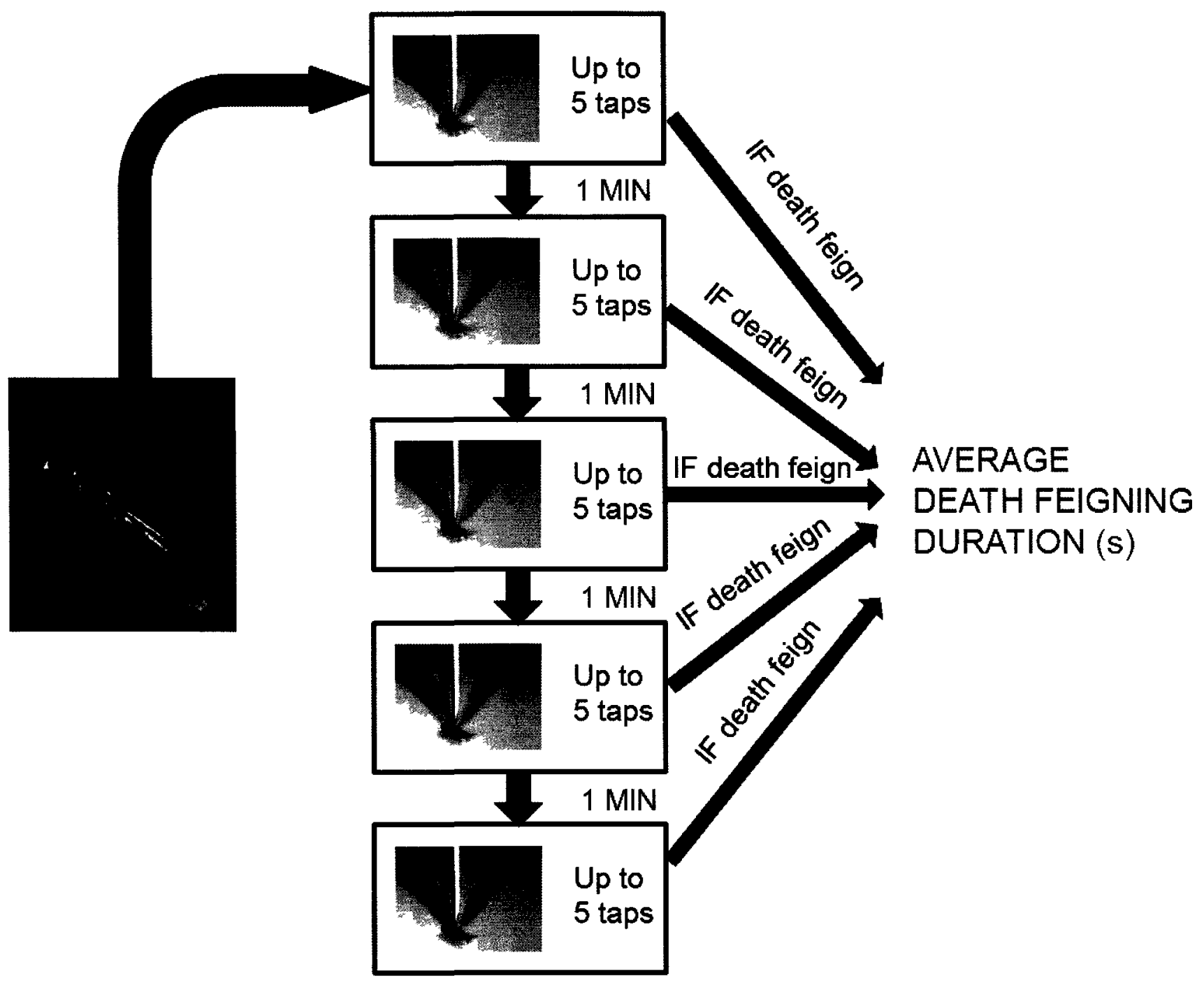

Figure 1. Illustration of the method for obtaining the average death feigning duration for comparison with chemical concentrations. 
The column (used a Phenomenex Zebron ZB-5ms column (30m long, $0.25 \mathrm{~mm}$ I.D. and 0.25 micron film thickness)) was held at $70^{\circ} \mathrm{C}$ for the first 3 minutes before increasing to a maximum temperature of $260^{\circ} \mathrm{C}$ at a rate of $15^{\circ} \mathrm{C}$ per minute. The SPME fibre was removed after four minutes and the sample was allowed to run for 14 minutes.

The second method of measuring chemical concentration was a hexane rinse. The same individuals that were tested with the SPME fibres were used in the hexane rinse tests, which were performed at least a day after the SPME fibre rub tests. Individuals were dropped into $1.0 \mathrm{ml}$ amber vials with glass inserts filled with $100 \mu$ of hexane. Vials were vortexed for one hour. After the vortexing, individuals (now deceased) were removed from the vials and the vials were stored in a freezer at $-4^{\circ} \mathrm{C}$ until they were tested. Vial testing was done over a two week period. $1 \mu$ of sample was picked up with a syringe and inserted into the syringe holder of an Agilent $6890 \mathrm{GC}$ coupled to a 5973 mass selective detector using helium as a carrier gas. The column (used a Phenomenex Zebron ZB-5ms column (30m long, 0.25mm I.D. and 0.25 micron film thickness)) was held at $70^{\circ} \mathrm{C}$ for the first 3 minutes before increasing to a maximum temperature of $260^{\circ} \mathrm{C}$ at a rate of $15^{\circ} \mathrm{C}$ per minute. A 4 minute solvent delay was applied to ensure that hexane was not picked up on the GC. Samples were run for 25 minutes.

Chemical concentrations were taken as the peak area from the $\mathrm{GC}$ plots generated for each sample. GC plots were analysed and chemical concentrations were extracted using Agilent MSD Chemstation D.01.02.16. MBQ, EBQ and PENT all had consistent retention times at $5.61 \mathrm{mins}, 6.83 \mathrm{mins}$ and $10.73 \mathrm{mins}$ respectively. 
$\underline{\text { Statistical analysis }}$

All statistical analyses were performed with SPSS 17.0. For the SPME rubbed samples, a large number of individuals did not emit any or all of the chemicals $(n=54$, proportion $=$ $45.0 \%$ ). As a result of a lack of normality, a Spearman's rank test was performed to test the relationship between average death feigning duration and chemical concentration. All the individuals that did not emit any chemicals or all of the chemical components were removed in complementary analyses, to see if there was any relation when only individuals emitting chemicals were examined. Chemical concentrations were transformed to meet the requirements of parametric testing (LN transformed MBQ and EBQ values, square root transformed PENT values) and a MANOVA was performed. Included factors in the analysis were sex, weight, sample group (Group1 and Group2), first attempt taps (the number of taps required to induce death feigning on the first attempt) and average death feigning duration. A PCA, on the three different chemicals, was performed on all individuals, and on only those individuals that emitted all three chemicals. The PCA was performed to combine the three chemical concentrations into one value, representing a more holistic measure of chemical defence based on independent components, so that a more direct analysis of the relationship between death feigning and chemical defence could be performed. Separate rank transformed ANOVAs were performed on both PCA analyses using the same factors as above.

For the hexane rinsed samples, 8 individuals ( 4 male and 4 female) were excluded because of damage to the vials. A MANOVA was run for all three chemical concentrations, using the same factors as above. A PCA analysis was performed on all the individuals, and an ANOVA was applied to the PCA analysis using the same factors as above. 
I also examined the relationship between SPME rub and hexane rinse values for all the individuals. I ran two sets of analyses; one that included all individuals, and one that excluded individuals that did emit all of the chemicals in the rubs. For each set, I used separate ANOVAs to compare the hexane rinse and SPME rub concentrations for each chemical separately. I also performed separate PCA analyses on the hexane rinsed and SPME rub concentrations, and analysed those with an ANOVA. Factors used for all ANOVAs were the same factors as above. 


\section{$\underline{\text { Results }}$}

\section{SPME rub samples}

There was no significant relationship between any of the chemical components and the average duration of death feigning when both individuals who emitted chemicals and those that did not, were included (MBQ: $\rho=0.091, N=120, P=0.322$; $E B Q: \rho=0.086, N=120, P=0.350$; PENT: $\rho=0.079, \mathrm{~N}=120, \mathrm{P}=0.393$ ). When individuals that did not emit all chemicals were excluded, there was still no significant relationship between chemical components and the average duration of death feigning $\left(\mathrm{MBQ}: \mathrm{R}^{2}=0.01, \mathrm{~F}_{1,65}=0.816, \mathrm{P}=0.370 ; \mathrm{EBQ}: \mathrm{R}^{2}=0.008\right.$, $\mathrm{F}_{1,65}=0.385, \mathrm{P}=0.537 ; \mathrm{PENT}: \mathrm{R}^{2}=0.005, \mathrm{~F}_{1,65}=0.051, \mathrm{P}=0.823$; Fig.2). There was a significant difference in chemical concentration between sexes for PENT $\left(F_{1,65}=4.212, P=0.045\right)$, with males producing more PENT than females.

After performing a PCA on the different chemical components for all individuals (first component of PCA explained $90.6 \%$ of variance), I found no significant relationship between chemical defence concentration and average death feigning duration $\left(\mathrm{R}^{2}=0.001, \mathrm{~F}_{1,119}=1.454\right.$, $\mathrm{P}=0.231$ ). However, there was a significant difference between the sexes for chemical defence concentration $\left(\mathrm{F}_{1,119}=5.318, \mathrm{P}=0.023\right)$, with males producing a higher concentration of chemicals than females. I performed an additional PCA, removing individuals who did not emit all three chemicals (first component of PCA explained 89.8 of variance), and I found no significant relationship between chemical defence concentration and average death feigning duration $\left(\mathrm{R}^{2}=0.012, \mathrm{~F}_{1,65}=0.353, \mathrm{P}=0.555\right.$; Fig.3). However, there was again a significant difference between the sexes $\left(F_{1,65}=4.126, P=0.047\right)$, with males producing a higher concentration of chemicals than females. 

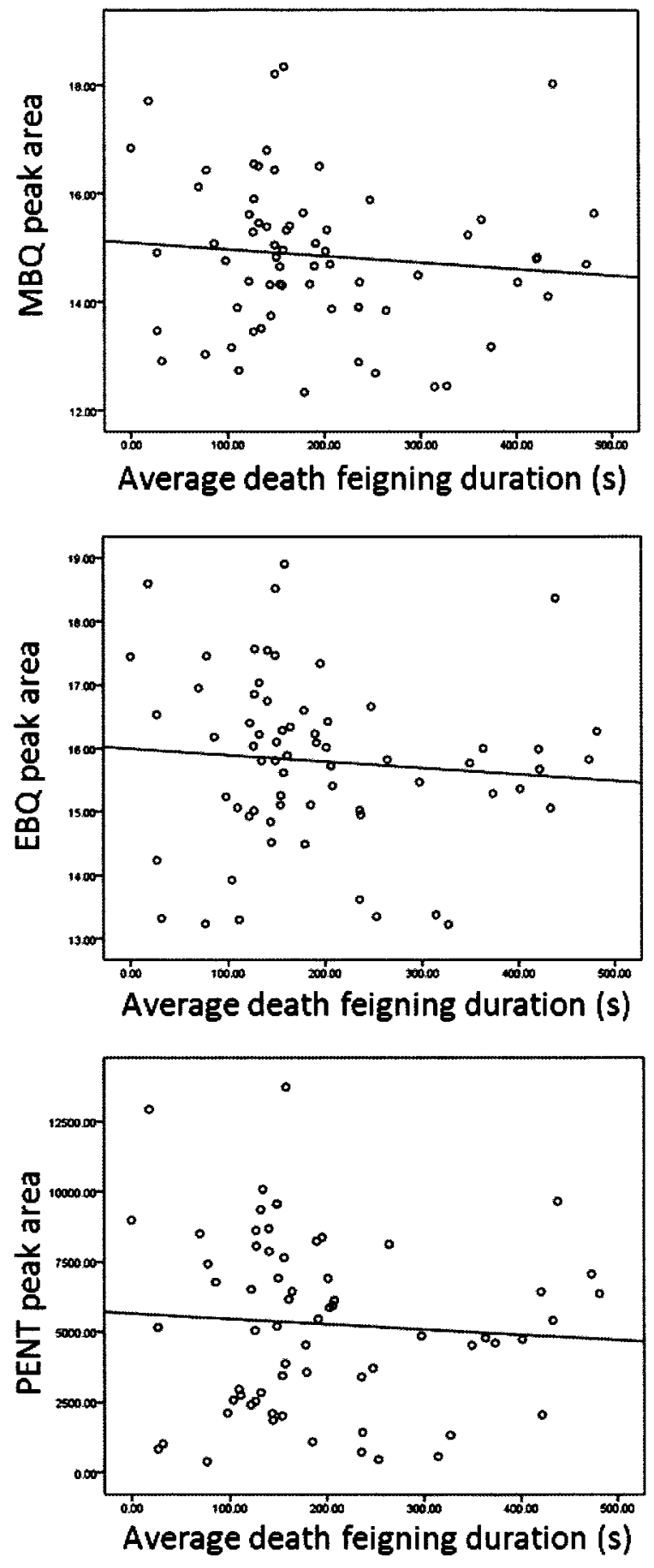

Figure 2. The relationships between chemical concentration (peak area of a GC plot) and the average death feigning duration (measured in seconds). The individuals who did not emit any chemical were excluded. From top to bottom: MBQ (LN transformed), EBQ (LN transformed), PENT (square root transformed). 


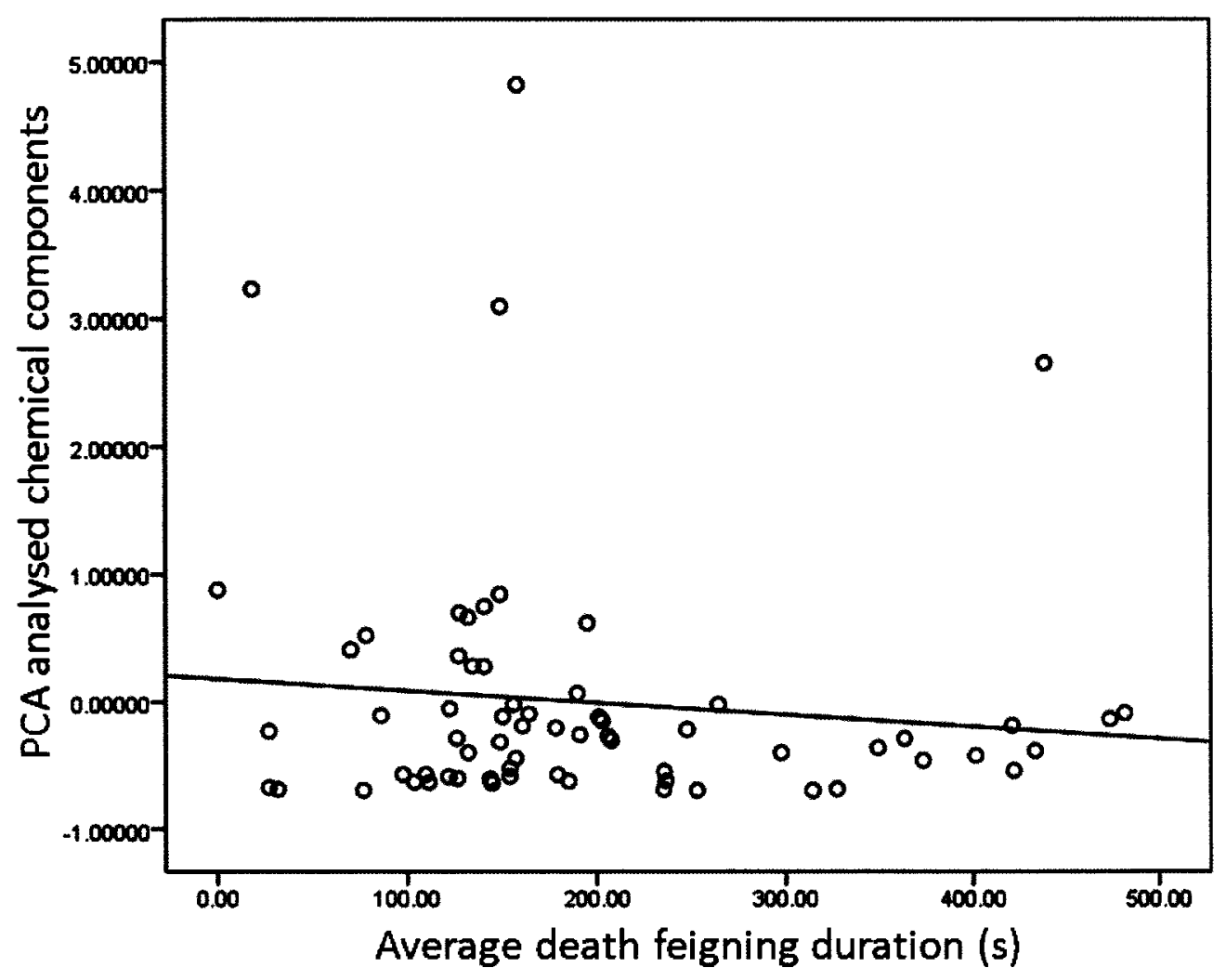

Figure 3. The relationship between the PCA of all the chemical components from the rubbed trials (only individuals who emitted all chemicals) and the average death feigning duration (measured in seconds). 


\section{Hexane rinse samples}

When examining the relationship between the three primary chemical components and the average duration of death feigning, I found no significant relationship between any of the chemicals and the average duration of death feigning $\left(\mathrm{MBQ}: \mathrm{R}^{2}=0.003, \mathrm{~F}_{1,111}=0.182, \mathrm{P}=0.671\right.$; EBQ: $\mathrm{R}^{2}=0.005, \mathrm{~F}_{1,111}=0.017, \mathrm{P}=0.898$; $\mathrm{PENT}: \mathrm{R}^{2}=0.021, \mathrm{~F}_{1,111}=2.745, \mathrm{P}=0.101$; Fig.4). There was a significant difference between the sexes for chemical concentrations, with males having greater concentrations than females for $\operatorname{EBQ}\left(\mathrm{F}_{1,111}=9.010, \mathrm{P}=0.003\right)$ and $\operatorname{PENT}\left(\mathrm{F}_{1,111}=12.035\right.$, $\mathrm{P}=0.001$ ). There was a significant positive relationship between chemical concentration and individual beetle weight for PENT $\left(\mathrm{R}^{2}=0.018, \mathrm{~F}_{1,111}=7.423, \mathrm{P}=0.008\right)$.

After performing a PCA on the different chemical components (first component of PCA explained $86.3 \%$ of variance), I found no significant difference between chemical defence and the average duration of death feigning $\left(\mathrm{R}^{2}=0.003, \mathrm{~F}_{1,111}=0.173, \mathrm{P}=0.678 ;\right.$ Fig.5). There was a significant difference in chemical concentration between the sexes $\left(\mathrm{F}_{1,111}=7.906, \mathrm{P}=0.006\right)$, with males having a higher concentration than females. There was also a significant positive relationship between chemical concentration and weight $\left(\mathrm{R}^{2}=0.018, \mathrm{~F}_{1,111}=4.403, \mathrm{P}=0.038\right)$. 

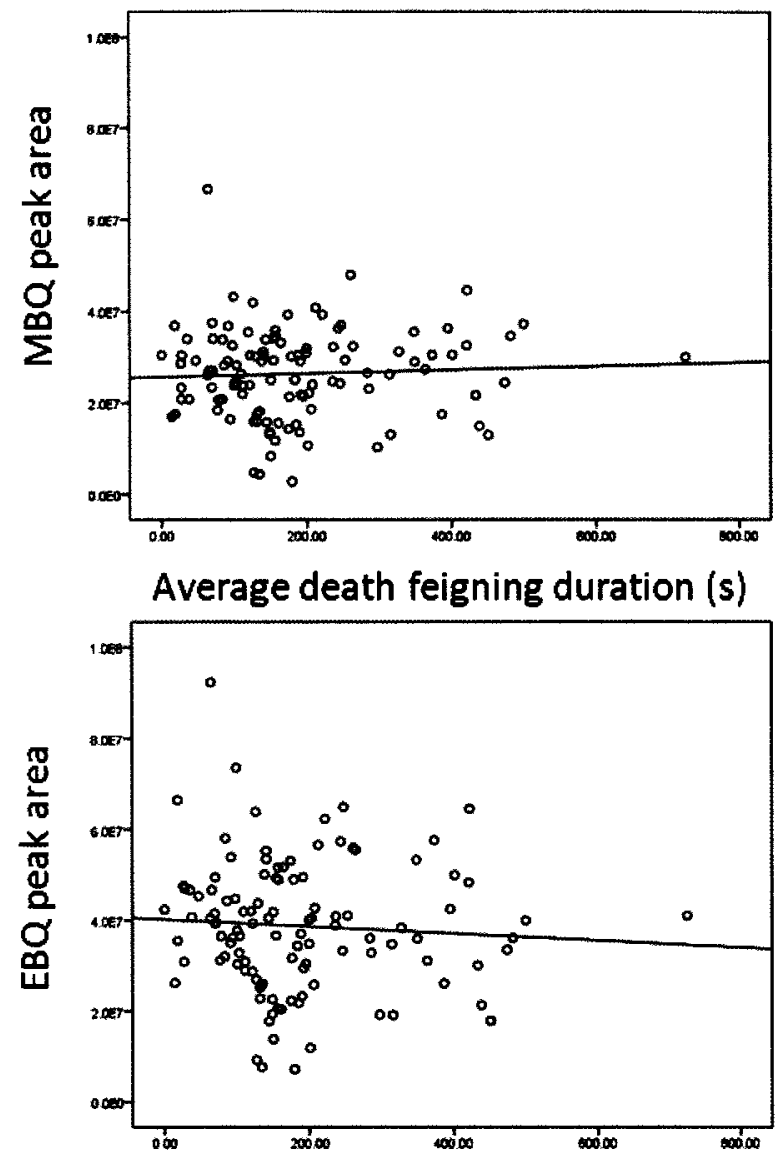

Average death feigning duration ( $s$ )

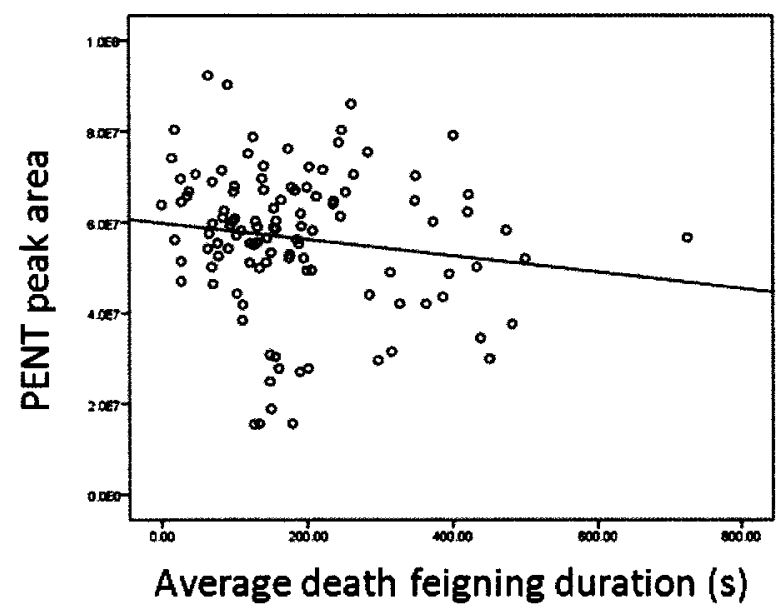

Figure 4. The relationship between the hexane rinsed chemical concentrations (peak area of a GC plot) and the average death feigning duration (measured in seconds). From top to bottom: MBQ, EBQ, PENT. 


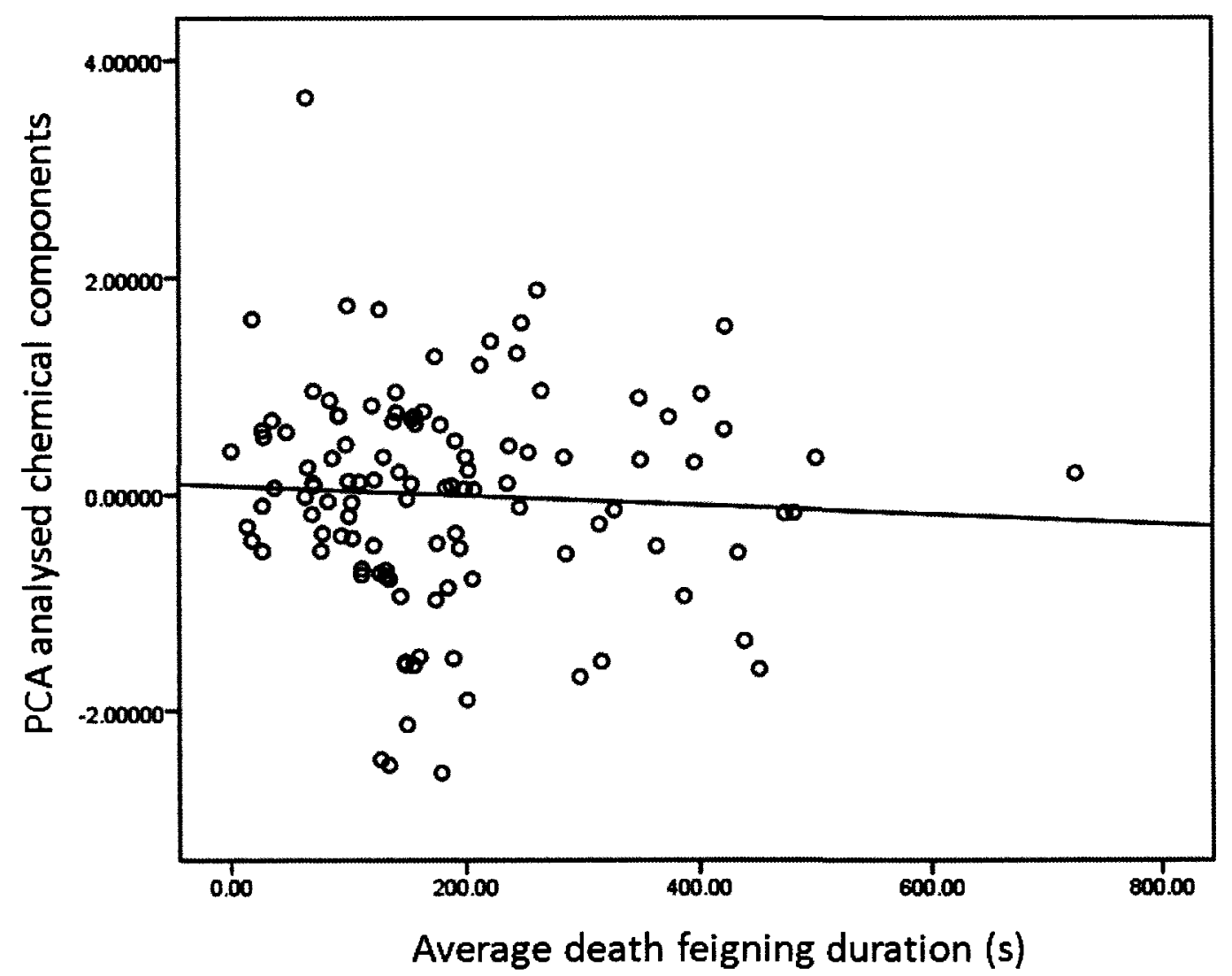

Figure 5. The relationship between the PCA of all the chemical components from the hexane rinsed trials and the average death feigning duration (measured in seconds). 


\section{Hexane rinse vs. SPME rub}

When all individuals were included, there was a significant negative relationship between the hexane rinse and SPME rub chemical concentrations for each individual chemical (MBQ: $\mathrm{R}^{2}=0.037, \mathrm{~F}_{1,111}=4.772, \mathrm{P}=0.031 ; \mathrm{EBQ}: \mathrm{R}^{2}=0.021, \mathrm{~F}_{1,111}=5.197, \mathrm{P}=0.025 ; \mathrm{PENT}: \mathrm{R}^{2}=0.097$, $\mathrm{F}_{1,111}=26.529, \mathrm{P}<0.001 ;$ Fig.6) and for all chemicals combined $\left(\mathrm{R}^{2}=0.065, \mathrm{~F}_{1,111}=12.791\right.$, $\mathrm{P}=0.001 ;$ Fig. 7 ).

When individuals that did not emit all chemicals were excluded from the analysis, only PENT displayed a significant negative relationship between the hexane rinse and the SPME rub chemical concentrations $\left(\mathrm{R}^{2}=0.047, \mathrm{~F}_{1,60}=9.668, \mathrm{P}=0.003\right)$. When all the chemicals were combined with PCA, there was no significant relationship between SPME rub and hexane rinse samples $\left(\mathrm{R}^{2}=0.034, \mathrm{~F}_{1,60}=3.338, \mathrm{P}=0.074\right)$. 

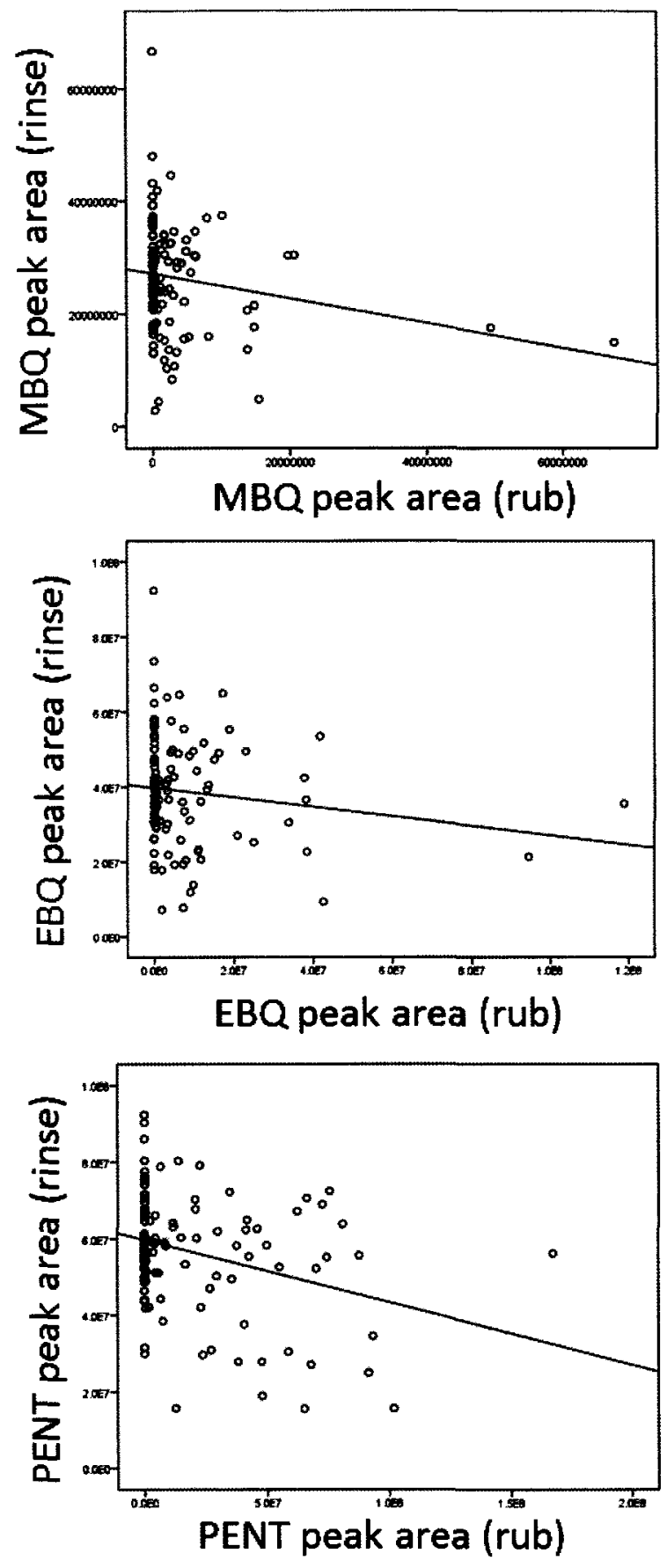

Figure 6. Relationships between the rinse chemical concentrations and the rub chemical concentrations for all individuals. From top to bottom: MBQ, EBQ, PENT. 


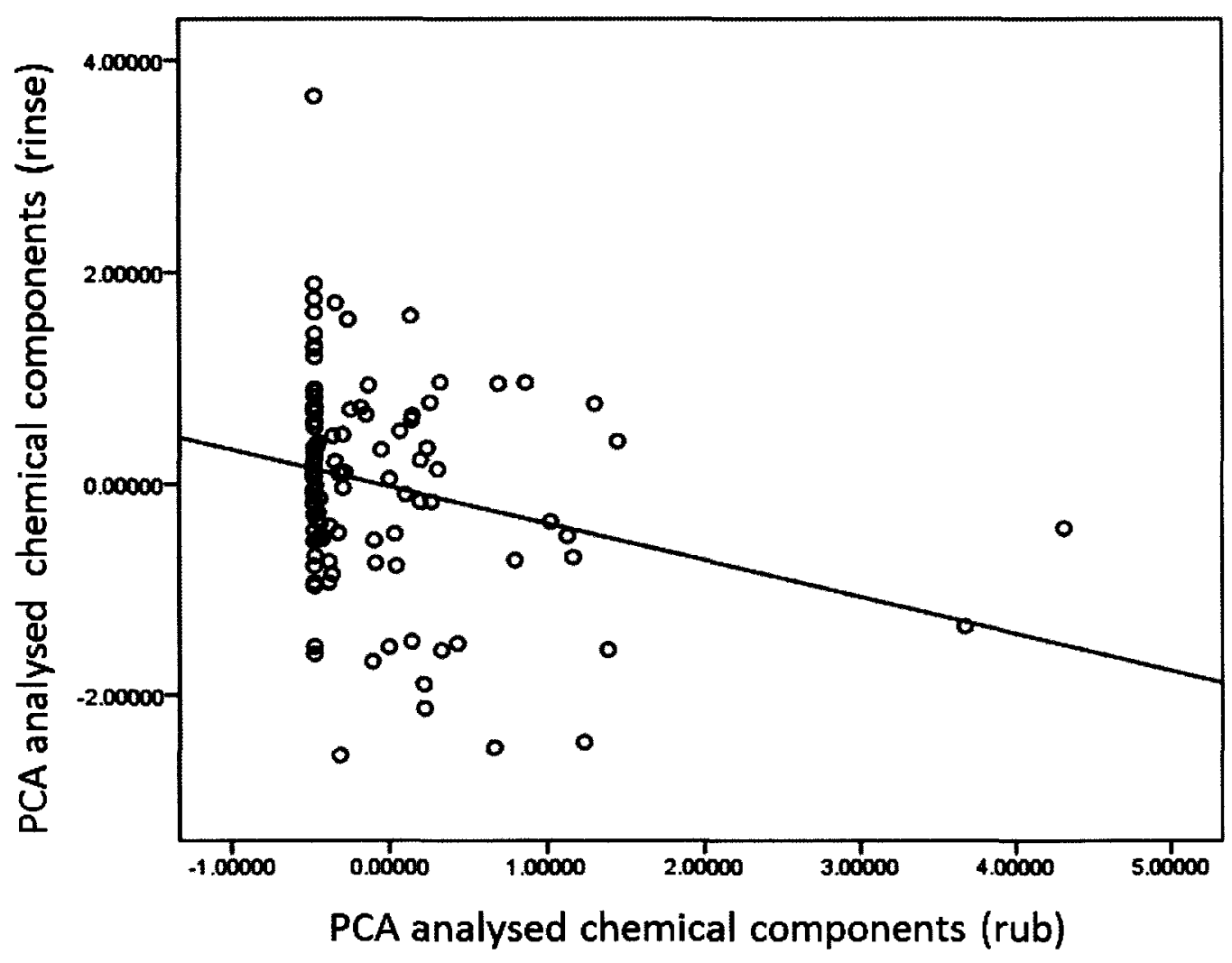

Figure 7. Relationship between the PCA analysed rinse samples and the PCA analysed rub samples for all individuals. 


\section{$\underline{\text { Discussion }}$}

The honest signal hypothesis for death feigning posits that individuals with a chemical defence will death feign as a means of signalling to predators that they are chemically defended and are unsuitable for consumption. Individuals that death feign would be expected to produce and emit a higher quantity or greater concentrated defensive secretion than those individuals who do not death feign. Alternatively, if a population has a high propensity to death feign, defensive chemical concentration would be expected to be positively correlated with death feigning duration.

I tested this hypothesis in the red flour beetle, $T$. castaneum. I examined the relationship between three primary chemical components that make up the $T$. castaneum defensive secretion, MBQ, EBQ and PENT, and the average death feigning duration. I found no significant relationship between any of the three chemical components and the average death feigning duration. These results are consistent with a previous study that showed no significant difference in MBQ concentration between death feigning and non-death feigning $T$. castaneum individuals (Miyatake et al. 2009). Overall given these results, it is unlikely that death feigning is an honest signal for chemical defence in $T$. castaneum in the manner proposed. Of course, while these results allow us to rule out the honest signal hypothesis for $T$. castaneum, it remains a potential hypothesis for death feigning behaviour in other species that death feign and use a chemical defence.

The positive relationship found between weight and chemical concentration is to be expected, as larger individuals should have a greater concentration of chemical because they produce more. However, the difference between sexes, with males having higher concentrations 
of chemicals than females is contrary to a previous study (Unruh et al. 1998). I investigated whether differences in weight between the sexes could be the reason for differences in chemical concentration. I found that females weighed significantly more than males, and both groups showed a trend of increasing chemical concentration with increasing weight but males tended to produce higher concentrated chemical than females of a similar weight. Another reason for this difference in chemical concentrations between the sexes may be due to using different populations for testing (myself and Unruh et al. (2008)), and that differences between the sexes vary between populations.

The results of comparing the hexane rinse concentrations and the SPME rub concentrations were unexpected. One would expect a positive linear trend between tests, with high concentration and low concentration individuals remaining consistent between tests. However, I found the reverse trend when all individuals were included, and no trend when those individuals that did not produce chemicals were removed (with the exception of a negative trend for PENT). One reason for this could be that the hexane rinse tests capture all the chemical that is released, while the SPME rub tests only capture residual chemical that may still be present on the individual. Another reason could be that a day between the rub and rinse tests was not enough to allow for individuals to refill chemical defence reserves and as a result, individuals that emitted all chemicals during the rub tests would be more likely to emit lower chemical levels in the rinse tests. As a result of this test, as well as the large proportion of individuals that did not produce all chemicals (45\%), I feel that the SPME rub test is less useful for testing the relationship between chemical defence and death feigning, as it potentially fails to capture the presence of released chemical, and is not as precise as the hexane rinse test. 
Given that the honest signal hypothesis for death feigning in $T$. castaneum is unlikely, there are two remaining hypotheses for death feigning as a phenomenon that hold some promise (Miyatake et al. 2009). The first hypothesis is the idea of "motion blindness" on the part of the predator. It posits that the predators, being attracted to prey movement, will either not be able to see a death feigning individual or will lose interest in a death feigning individual. This is entirely consistent with Miyatake et al.'s $(2004,2009)$ observation that those individuals that death feigned survived, while those that did not were subsequently eaten by the spiders, which hunt according to a chase and kill method (Miyatake et al. 2004, 2009).

The second hypothesis has been labelled as the "selfish" hypothesis, which posits that death feigners are successful because of the presence of non-death feigners that are in close proximity and that distract the predator from the death feigning individuals (Miyatake et al. 2009) - thus, the phenomenon is one of two strategies whose success is frequency dependent. There is evidence to support that death feigners live longer than non-death feigners when grouped together with a predator than when the two types were kept separately (Miyatake et al. 2009). Intriguingly, there is evidence that non-death feigners move more quickly than death feigners (Miyatake et al. 2008b), which produces the converse explanation, that not deathfeigning is an honest signal of running speed, and suggests that individuals may chose the defensive strategy that maximises their survivorship. More research is clearly required to tease apart the two preceding hypotheses, since both rest on motion-blindness but the latter assumes frequency-dependence while the former does not.

This study also raises the issue of why individuals would have both chemical defence and death feigning. Not all predators will exhibit motion blindness and not all predators will be 
deterred by chemical defence, so the evolved defenses observed in Tribolium could be an evolved response to an environment with multiple predators (Endler and Mappes 2004). However, to evaluate this explanation more research into the natural history of $T$. castaneum is required, specifically their natural predators, as well as how they respond in lab to multiple predators and in different mixed samples of death feigners and non-death feigners.

While there is no evidence to support the honest signal hypothesis for death feigning in $T$. castaneum, there is still room for further study on the matter. There is recent evidence that shows differences in chemical concentration between individuals in relation to population density, with those individuals reared in a high density environment producing greater amounts of MBQ and EBQ than those individuals reared in a low density environment (Duehl et al. 2011). It would be interesting to see if there was any relationship between death feigning duration and chemical defence when compared between densities. Furthermore, as I am not aware of any work done examining differences in death feigning behaviour between individuals reared in different population densities, this type of research would answer two questions at once. In addition to this research, it would also be interesting to examine if there is any relationship between 4,8 dimethyldecanal (DMD), a T. castaneum aggregation pheromone that may be used in mating (Bloch Qazi et al. 1998; Arnaud et al. 2002), and death feigning duration. One could expect to see variations in DMD between death feigners and non-death feigners, as there is evidence that shows death feigners mating less than non-death feigners (Nakayama and Miyatake 2009).

In conclusion, I found no evidence to support the honest signal hypothesis for death feigning in T. castaneum, having found no relationship between their chemical defence and average death feigning duration. While there are at least two additional hypotheses that may 
explain death feigning in T. castaneum, predator motion blindness and relying on predator distraction by conspecifics, additional research is required to test and discriminate between these hypotheses. 


\section{Chapter 4-General conclusions}

In the first chapter, I briefly reviewed the current literature on death feigning. Death feigning is a secondary anti-predator defence wherein individuals enter a state of immobility after contact with a predator or in life-threating situations. Death feigning is widespread, being exhibited by numerous species across taxa. While death feigning has been noted in the literature for some time, research into the ultimate causes of death feigning is a nascent and ongoing area of research. There are several different hypotheses that attempt to explain why individuals death feign, however, there is very little evidence to support (or reject) most of these hypotheses. One of these hypotheses posits that death feigning is related to chemical defence in some species, with death feigning acting as an honest signal of toxicity to predators. The goal of my thesis was to examine if this hypothesis proved to be an acceptable explanation for death feigning behaviour in Tribolium castaneum. However, before I could answer this question, I had to investigate the repeatability and variability of death feigning behaviour in $T$. castaneum, research that would allow me to properly classify the degree of death feigning in individuals.

In the second chapter, I investigated the repeatability and variability of death feigning behaviour in T. castaneum individuals. As there was some evidence of death feigning being a polymorphic trait in T. castaneum (Prohammer and Wade 1981), before I could examine the relationship between death feigning and chemical defence, I had to examine whether individuals could be dichotomously classified as death feigners and non-death feigners, or whether individuals would be classified along a continuous spectrum from low to high degrees of death feigning. Using three different populations of $T$. castaneum, I surveyed death feigning behaviour in individuals who were exposed to a perceived threat (metal tweezers), used to induce death feigning, five times in quick succession within a single day (short term trials) or once in a day for 
five days (medium term trials). I found that individuals were more likely to death feign with successive attempts (short and medium term), and individuals death feigned longer with each successive attempt (short and medium term, with the exception of the Croatia population in the medium term). I found differences in death feigning duration among populations and (for the first time) between sexes (females death feigned longer than males). Additionally, I found that individuals that death more often or that require less provocation to death feign will death feign for longer, on average, than individuals that are less likely to death feign or require more provocation to death feign. Finally, I found that death feigning duration between days is not very repeatable but that short term trends of increasing duration are consistent between individuals. As a result of this study, I concluded that individuals could not simply be classified as death feigners and non-death feigners, and that using the average death feigning duration taken from the multiple attempts of the short term trials would be the most useful indicator of degrees of death feigning for examining the relationship between chemical defence and death feigning in $T$. castaneum.

In the third chapter, I examined whether a relationship existed between chemical defence and death feigning in T. castaneum. Previous research had led to speculation that death feigning may act as an honest signal of toxicity (Miyatake et al. 2004; Ruxton 2006). This relationship had been investigated in T. castaneum previously, with no evidence of a relationship between death feigning and chemical defence (Miyatake et al. 2009). However, researchers only examined one of three primary chemicals that make up the $T$. castaneum defensive secretion (methyl-1,4-benzoquinone (MBQ), which is the component lowest in concentration), as well as only looking at males. I felt that an examination of all three chemical components (MBQ, ethyl1,4-benzoquinone (EBQ), and 1-pentadecene (PENT)), as well as examining both sexes would 
provide for a more adequate study of the honest signal hypothesis for death feigning in $T$.

castaneum. Based on work from the previous chapter, I used the average death feigning duration calculated from five death feigning attempts in one day (short term trials), as a measure of death feigning. Using two different methods of chemical concentration capture (SPME fibre rub and hexane rinse) and analysis using a gas chromatography-mass spectrometer (GC-MS), I found no relationship between any of the three primary chemical components and average death feigning duration. This research provides further evidence that death feigning in T. castaneum is probably not acting as an honest signal of chemical toxicity, although this hypothesis may still find validation in other species that death feign and have a chemical defence.

While there is no evidence to support the hypothesis that death feigning is an honest signal of toxicity in T. castaneum, there is further work that could be done. Recent research by Duehl et al. (2011) has shown variations in the release of defense secretions (MBQ and EBQ) based on the density of individuals. It would be interesting to examine if there is a relationship between chemical concentration and death feigning duration in individuals that have been kept in groups, as opposed to separated. Additionally, it would be interesting to investigate if there are any differences in death feigning behaviour between individuals that have been reared in isolation, as opposed to those reared in groups with other individuals. Also, as I am not aware of any research that has been done on the effect of rearing density on death feigning behaviour, this would provide a new area of research for death feigning. It would also be interesting to test for any relationship between death feigning duration and 4,8-dimethyldecanal (DMD), which is an aggregation pheromone that may be used by T. castaneum for mating (Bloch Qazi et al. 1998; Arnaud et al. 2002). As there is previous evidence for differences in mating levels between death feigning and non-death feigning individuals (Nakayama and Miyatake 2009), one might expect 
there to be a difference in DMD levels between individuals with a higher degree of death feigning and those with a lower degree of death feigning.

With evidence lacking for death feigning as an honest signal of toxicity, there are two other hypotheses that may explain death feigning in T. castaneum: 1) Motion blindness by the predator, wherein motionless prey do not attract predators that are active hunters, and 2) Predator distraction by non-death feigning prey, wherein an individual who is death feigning survives because the predator leaves the individual alone to attack another individual that is moving. There is evidence to support the first hypothesis and some evidence to support the second hypothesis (Miyatake et al. 2004; Miyatake et al. 2009), however, research is needed to disentangle the two hypotheses and to verify whether it is one or the other or both working together. It seems likely that motion blindness by the predator works when the individual is alone, and that effect is amplified when there are other individuals moving about to distract the predator.

There is still a lot more research and work to do before we can gain a better understanding of the ultimate causes of death feigning, and why it is so widespread. I feel that my thesis has been a step forward, helping to further dispel one hypothesis, while highlighting additional hypotheses for death feigning in $T$. castaneum. I hope that through my research, experimentation, and presentations at the University of Alberta Mathematical Biology workshop (2010) and Canadian Society for Ecology and Evolution conference (2011), that I have raised awareness about death feigning, the current state of death feigning research, as well as future areas of research. 


\section{Appendix}

Intraclass correlation coefficient (ICC) is often used in biology as a measure of the repeatability of a phenotypic trait. It is used to explain the proportion of phenotypic variation between rather than within individuals (Lessells and Boag 1987; Nakagawa and Schielzeth 2010). In my thesis, I calculated ICC to ascertain how repeatable death feigning duration was in individuals between days in medium trials. I used SPSS 17.0, which calculates ICC values based on methods outlined by McGraw and Wong (1996). I calculated a two-way mixed ICC model, with conditions of absolute agreement, taking the single measure calculation. I used a two-way mixed model because individual was treated as a random factor and day was treated as a fixed factor. SPSS gives the option of choosing absolute agreement versus consistency. This choice boils down to whether or not the variation between days is important and should be included in the model (absolute agreement) or not (consistency). SPSS also outputs a single measure ICC and average measure ICC. I used single measure ICC values because I had individual measurements taken once a day for five days. If I was looking at average measurements of individuals or days, I would have used the average measure ICC. The two-way mixed effects ICC single measure value, with the absolute agreement condition was calculated with the following formula:

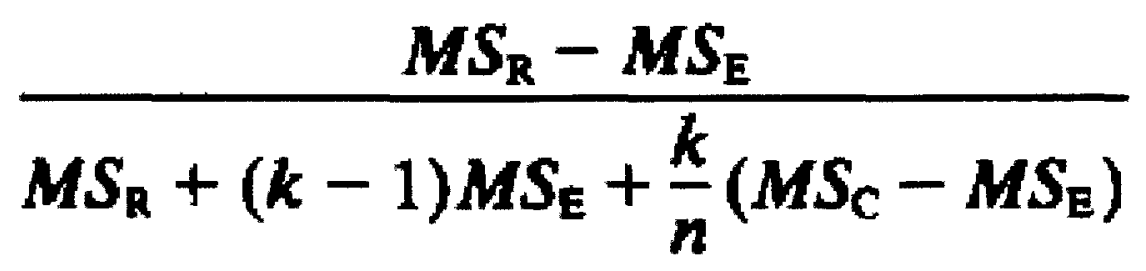


with $M S_{R}$ (mean square of rows (between individuals), $M S_{E}$ (mean square of error), and $M S_{C}$ (mean square of columns (between days)) all taken from an ANOVA, $k$ representing the number of measurements for each individual, and $n$ being the number of individuals sampled (McGraw and Wong 1996). 


\section{References}

Abrams, Murray Peter. Human tonic immobility: an exploration of three trauma contexts. MA thesis. The University of Regina, Regina, Saskatchewan. ProQuest Dissertations and Theses. Web. 26 June 2011.

Acheampong, S., and B.K. Mitchell. 1997. Quiescence in the Colorado potato beetle, Leptinotarsa decemlineata. Entomologia Experimentalis et Applicata. 82:83-89.

Arnaud, L., G. Lognay, M. Verscheure, L. Leenaers, C. Gaspar, and E. Haubruge. 2002. Is dimethydecanal a common aggregation pheromone of Tribolium flour beetles? Journal of Chemical Ecology. 28(3):523-532.

Bates, Doug. "[R] Imer, p-values and all that." 19 May 2006. Web. 26 June 2011. < https://stat.ethz.ch/pipermail/r-help/2006-May/094765.html>

Beux dos Santos, M., M.C.L.M. de Oliveira, L. Verrastro, and A.M. Tozetti. 2010. Playing dead to stay alive: death-feigning in Liolaemus occipitalis (Squamata: Liolaemidae). Biota Neotropica. 10:361-364

Bilde, T., C. Tuni, R. Elsayed, S. Pekar, and S. Toft. 2006. Death feigning in the face of sexual cannibalism. Biology Letters. 2:23-25.

Bloch Qazi, M.C., C.R.B. Boake, and S.M. Lewis. 1998. The femoral setiferous glands of Tribolium casntaneum males and production of the pheromone 4,8-dimethyldecanal.

Entomologia Experimentalis et Applicata. 89:313-317

Brodie, E.D., J.A. Johnson, and C.K. Dodd. 1974. Immobility as a defensive behaviour in salamanders. Herpetologica. 30:79-85.

Bryson, B. 1998. A walk in the woods: Rediscovering America on the Appalachian trail. New York: Harper Collins Publishers.

Cantor, C. 2005. Evolution and posttraumatic stress: Disorders of vigilance and defence. New York: Routledge Publishing.

Caro, T. 2005. Antipredator defenses in birds and mammals. Chicago: The University of Chicago Press.

Cassill, D.L., K. Vo, and B. Becker. 2008. Young fire ant workers feign death and survive aggressive neighbours. Naturwissenschaften. 95:617-624.

Couturier, M., J.B. Hicks, D. Rouison, and Y. Pelletier. 2005. Thermal initiation of thanatosis to improve the pneumatic removal of the Colorado potato beetle. Canadian Biosystems Engineering. 47:2.5-2.12. 
Darwin, C. 1883. A posthumous essay on instinct. Taken from Mental evolution in animals; Romanes, G. 1970. Westmead: Gregg International Publishers Limited.

Duehl, A.J., R.T. Arbogast, and P.E.A. Teal. 2011. Density-related volatile emissions and responses in the red flour beetle, Tribolium castaneum. Journal of Chemical Ecology. 37:525532.

Edmunds, M. 1974. Defence in animals. New York: Longman Group Limited.

Edmunds, M. 1976. The defensive behaviour of Ghanaian praying mantids with a discussion of territoriality. Zoological Journal of the Linnaean Society. 58:1-37.

Endler, J.A., and J. Mappes. 2004. Predator mixes and the conspicuousness of aposematic signals. The American Naturalist. 163(4):532-547.

Fleming, P.A.. D. Muller, and P.W. Bateman. 2007. Leave it all behind: a taxonomic perspective of autotomy in invertebrates. Biological reviews. 82(3):481-510.

Forkman, B., A. Boissy, M.C. Meunier-Salaün, E. Canali, and R.B. Jones. 2007. A critical review of fear tests used on cattle, pigs, sheep, poultry and horses. Physiology \& Behavior. 92:340-374.

Francq, E.N. 1969. Behavioral aspects of feigned death in the Opossum Didelphis marsupialis. American Midland Naturalist. 81:556-568.

Frost, S.W. 1942. Insect life and insect natural history. New York: Dover Publications, Inc.

Fu, X., O. Nobuyoshi, V.B. Meyer-Rochow, Y. Wang, and C. Lei. 2006. Reflex-bleeding in the firefly Pyrocoelia pectoralis (Coleoptera: Lampyridae): Morphological basis and possible function. The Coleopterists Bulletin. 60:207-215.

Fu, X., F.V. Vencl, O. Nobuyoshi, V.B. Meyer-Rochow, C. Lei, and Z. Zhang. 2007. Structure and function of the eversible glands of the aquatic firefly Luciola lei (Coleoptera: Lampyridae). Chemoecology. 17:117-124.

Gerald, G.W. 2008. Feign versus flight: influences of temperature, body size and locomotor abilities on death feigning in neonate snakes. Animal Behaviour. 75:647-654.

Gregory, P.T., L.A. Issac, and R.A. Griffiths. 2007. Death feigning by grass snakes (Natrix natrix) in response to handling by human "Predators". Journal of Comparative Psychology. 121:123-129.

Gyssels, F.G.M., and R. Stoks. 2005. Threat-sensitive responses to predator attacks in a damselfly. Ethology. 111:411-423. 
Hansen, L.S., S.F. Gonzalez, S. Toft, and T. Bilde. 2008. Thanatosis as an adaptive male mating strategy in the nuptial gift-giving spider Pisaura mirabilis. Behavioral Ecology. 165:546-551.

Happ, G.M. 1968. Quinone and hydrocarbon production in the defensive glands of Eleodes longicollis and Tribolium castaneum (Coleoptera, Tenebrionidae). Journal of Insect Physiology. 14(12):1821-1837.

Hennig, C.W., and W.P. Dunlap. 1977. Circadian rhythms and the effects of lighting on tonic immobility in two species of lizard (Anolis carolinensis and Hemidactylus turcicus). Behavioral Biology. 20:523-528.

Holmes, S.J. 1916. Studies in animal behaviour. Boston: The Gorham Press.

Honma, A., S. Oku, and T. Nishida. 2006. Adaptive significance of death feigning posture as a specialized inducible defence against gape-limited predators. Proceeding of the Royal Society B. 273:1631-1636.

Howe, J. 1991. Field observations of death feigning in the convict tang, Acanthurus triostegus (Linnaeus), with comments on the nocturnal color pattern in juvenile specimens. Journal of Aquariculture and Aquatic Sciences. 6:13-15.

Hozumi, N., and T. Miyatake. 2005. Body-size dependent difference in death-feigning behaviour of adult Callosobruchus chinensis. Journal of Insect Behavior. 18:557-566.

Jones, R.B. 1977. Repeated exposure of the domestic chick to a novel environment: effects on behavioural responses. Behavioural Processes. 2:163-173.

Jones, R.B. 1988. Repeatability of fear ranks among adult laying hens. Applied Animal Behaviour Science. 19:297-304

Kuriwada, T., N. Kumano, K. Shiromoto, and D. Haraguchi. 2009. Copulation reduces the duration of death-feigning behaviour in the sweetpotato weevil, Cylas formicarius. Animal Behaviour. 78:1145-1151.

Kuriwada, T., N. Kumano, K. Shiromoto, and D. Haraguchi. 2010. Effect of irradiation on deathfeigning behaviour in the male sweetpotato weevil Cylas formicarius (Coleoptera: Blentidae). Florida Entomologist. 93(1):39-44.

Lefebvre, L., and M. Sabourin. 1977. Effects of spaced and massed repeated elicitation on tonic immobility in the goldfish (Carassius auratus). Behavioral Biology. 21:300-305.

Lessells, C.M. and P.T. Boag. 1987. Unrepeatable repeatabilities: a common mistake. The Auk. 104(1):116-121. 
Marks, I.M. 1987. Fears, phobias, and rituals: panic, anxiety, and their disorders. New York: Oxford University Press, Inc.

McGraw, K.O., and S.P. Wong. 1996. Forming inferences about some intraclass correlation coefficients. Psychological Methods. 1(1):30-46

McKaye, K.R. 1981. Field observation on death feigning: a unique hunting behaviour by the predatory cichlid, Haplochromis livinstoni, of Lake Malawi. Environmental Biology of Fish. 6:361-365.

Milewski, Terry. "Montreal actors uses film knowledge to escape Mumbai bloodbath." CBC News. November 30 2008. Web. June 26 2011. <www.cbc.ca/news/canada/montreal/story $\underline{12008 / 11 / 30 / \text { f-rudder.html }>}$

Miyatake, T. 2001. Diurnal periodicity of death-feigning in Cylas formicarius (Coleoptera: Brentidae). Journal of Insect Behavior. 14:421-432.

Miyatake, T. 2001b. Effects of starvation on death-feigning in adults of Cylas formicarius (Coleoptera:Brentidae). Annals of the Entomological Society of America. 94:612-616.

Miyatake, T., K. Katayama, Y. Takeda, A. Nakashima, A. Sugita, and M. Mizumoto. 2004. Is death-feigning adaptive? Heritable variation in fitness difference of death-feigning behaviour. Proceedings of the Royal Society B. 271:2293-2296.

Miyatake, T., K. Okada, and T. Harano. 2008. Negative relationship between ambient temperature and death-feigning intensity in adult Callosobruchus maculates and Callosobruchus chinensis. Physiological Entomology. 33:83-88.

Miyatake, T., K. Tabuchi, K. Sasaki, K. Okada, K. Katayama, and S. Moriya. 2008b. Pleiotropic antipredator strategies, fleeing and feigning death, correlated with dopamine levels in Tribolium castaneum. Animal Behaviour. 75:113-121.

Miyatake, T., S. Nakayama, Y. Nishi, and S. Nakajima. 2009. Tonically immobilized selfish prey can survive by sacrificing others. Proceedings of the Royal Society B. 276(1668):27632767.

Nakagawa, S., and H. Schielzeth. 2010. Repeatability for Gaussian and non-Gaussian data: a practical guide for biologists. Biological Reviews. 85:935-956.

Nakayama, S., and T. Miyatake. 2009. Positive genetic correlations between life-history traits and death-feigning behaviour in adzuki bean beetle (Callosobruchus chinensis). Evolutionary Ecology. 23:711-722. 
Nakayama, S., Y. Nishi, and T. Miyatake. 2009. Genetic correlation between behavioural traits in relation to death-feigning behaviour. Population Ecology. 52(2):329-335.

Oakley, D.A., and H.C. Plotkin. 1977. Ontogeny of tonic immobility in the rabbit. Behavioral Biology. 19:64-75.

Ohno, T., and T. Miyatake. 2007. Drop or fly? Negative genetic correlation between deathfeigning intensity and flying ability as alternative anti-predator strategies. Proceedings of the Royal Society B. 274:555-560.

Prohammer, L.A., and M.J. Wade. 1981. Geographic and genetic variation in death-feigning behaviour in the flour beetle, Tribolium castaneum. Behavior Genetics. 11:395-401.

Rémignon, H., A.D. Mills, D. Guémené, V. Desrosiers, M. Garreau-Mills, M. Marche, and G. Marche. 1998. Meat quality traits and muscle characteristics in high or low fear lines of Japanese quails (Coturnix japonica) subjected to acute stress. British Poultry Science. 39(3):372-378.

Rouse, M.W., E.M. Nestor, C.J. Parot, and P.N. DeLand. 2004. A re-evaluation of the development eye movement (DEM) test's repeatability. Optometry \& Vision Science. 81:934938.

Ruxton, G. 2006. Grasshoppers don't play possum. Nature. 440:880.

Ruxton, G.D., T.N. Sherratt, and M.P. Speed. 2004. Avoiding attack: The evolutionary ecology of crypsis, warning signals and mimcry. Oxford: Oxford University Press.

Sargeant, A.B., and L.E. Eberhardt. 1975. Death feigning by ducks in response to predation by Red Foxes (Vulpes fulva). American Midland Naturalist. 94:108-119.

Sazima, I. 1974. Experimental predation on the leaf-frog Phyllomedusa rohdei by the water snake Liophis miliaris. Journal of Herpetology. 8(4):376-377.

Shaffer, H.B. 1978. Relative predation pressure on salamanders (Caudata:Plethodontidae) along an altitudinal transect in Guatemala. Copeia. 1978:268-272.

Sherratt, T.N., A. Rashed, and C.D. Beatty. 2004. The evolution of locomotory behaviour in profitable and unprofitable simulated prey. Oecologia. 138:143-150.

Srygley, R.B. 2004. The aerodynamic costs of warning signals in palatable mimetic butterflies and their distasteful models. Proceedings of the Royal Society B. 271(1539):589-594.

Toledo, L.F., I. Sazima, and C.F.B. Haddad. 2010. Is it all death feigning? Case in anurans. Journal of Natural History. 44:1979-1988. 
Unruh, L.M., R. Xu, and K.J. Kramer. 1998. Benzoquinone levels as a function of age and gender of the red flour beetle, Tribolium castaneum. Insect Biochemistry and Molecular Biology. 28:969-977.

Villaverde, M.L., M.P. Juárez, and S. Mijailovsky. 2007. Detection of Tribolium castaneum (Herbst) volatile defensive secretions by solid phase microextraction-capillary gas chromatography (SPME-CGC). Journal of Stored Products Research. 43:540-545.

Von Endt, D.W., and J.W. Wheeler. 1971. 1-Pentadecene production in Tribolium confusum. Science. 172:60-61.

Walter, D.E., and H.C. Proctor. 1998. Feeding behaviour and phylogeny: observations on early derivative Acari. Experimental and Applied Acarology. 22:39-50.

Watsky, M.A., and S.H. Gruber. 1990. Induction and duration of tonic immobility in the lemon shark, Negaprion brevirostris. Fish Physiology and Biochemistry. 8(3):207-210.

Whitman, P.A., J.A. Marshall, and E.C. Keller, Jr. 1986. Tonic immobility in the smooth dogfish shark, Mustelus canis (Pisces, Carcharhinidae). Copeia. 1986:829-832.

\section{Wikipedia images}

Convict tang - Zinkova, M. "Acanthurus triostegus and Labroides phthirophagus.jpg." 2008. Web. 27 Jun 2011. <http://ca.wikipedia.org/wiki/Fitxer:Acanthurus_triostegus_and_Labroides phthirophagus_.jpg $>$.

Grass snake - Viridliflavus, P.S. "BatrixNatrixBellyPattern.JPG." 28 Aug 2005. Web. 27 Jun 2011. <http://en.wikipedia.org/wiki/File:BatrixNatrixBellyPattern.JPG>.

Mallard duck - Garrett, G.S. "Mallard in flight.jpg." 15 Apr 2009. Web. 27 Jun 2011.

$<$ http://en.wikipedia.org/wiki/File:Mallard_in_flight.jpg>

Opossum - Pope, C. "Opossum 2.jpg." 21 Feb 2007. Web. 27 Jun 2011.

$<$ http://en.wikipedia.org/wiki/File:Opossum_2.jpg>.

Red flour beetle - Day, E. "Tribolium castaneum87-300.jpg." 2 Sept 2009. Web. 27 Jun 2011. $<$ http://en.wikipedia.org/wiki/File:Tribolium_castaneum87-300.jpg $>$.

Salamander - Alzate, E."Bolitoglossa phalarosoma.jpg." Web. 27 Jun 2011. $<$ http://commons.wikimedia.org/wiki/File:Bolitoglossa_phalarosoma.jpg>. 